\title{
Synaptic Localization and Activity of ADAM10 Regulate Excitatory Synapses through N-Cadherin Cleavage
}

\author{
Matteo Malinverno, ${ }^{1}$ Mario Carta, ${ }^{2}$ Roberta Epis, ${ }^{1,4}$ Elena Marcello, ${ }^{1}$ Chiara Verpelli, ${ }^{3}$ Flaminio Cattabeni, ${ }^{1}$ Carlo Sala, ${ }^{3}$ \\ Christophe Mulle, ${ }^{2}$ Monica Di Luca, ${ }^{1}$ and Fabrizio Gardoni ${ }^{1}$ \\ ${ }^{1}$ Department of Pharmacological Sciences, University of Milan, 20133 Milan, Italy, ${ }^{2}$ Laboratoire Physiologie Cellulaire de la Synapse, Centre National de la \\ Recherche Scientifique Unité Mixte de Recherche 5091, Bordeaux Neuroscience Institute, University of Bordeaux 2, 33077 Bordeaux, France, ${ }^{3}$ Consiglio \\ Nazionale delle Ricerche, Institute of Neuroscience, Cellular and Molecular Pharmacology, Department of Pharmacology, University of Milan, 20129 Milan, \\ Italy, and ${ }^{4}$ XigenPharma, 1003 Lausanne, Switzerland
}

$\mathrm{N}$-Cadherin has an important role during dendrite arborization, axon guidance, and synaptogenesis. In particular, at synaptic sites, $\mathrm{N}$-cadherin is involved in the regulation of cell-cell adhesion and in morphology and plasticity control. Recent studies have shown that N-cadherin can be cleaved by the metalloproteinase ADAM10. Here we demonstrate that impairing ADAM10 localization and activity at synaptic sites decreases its processing of $\mathrm{N}$-cadherin. This leads to an accumulation of the full-length form of $\mathrm{N}$-cadherin, to an increase in spine head width, and to modifications of the number and function of glutamate receptors of AMPA type, both in vitro and in vivo. Our results indicate a key role for ADAM10 in the complex sequence of events through which $\mathrm{N}$-cadherin affects spine maturation and controls structure and function of glutamatergic synapses.

\section{Introduction}

Glutamate released at excitatory synapses acts toward different types of ionotropic glutamate receptors (iGluRs): kainate, AMPA, and NMDA type (Dingledine et al., 1999; Nusser, 2000; Pinheiro and Mulle, 2006; Newpher and Ehlers, 2008). Clustering of iGluRs at synaptic sites and their subunit composition influence the synaptic response evoked by given stimuli (Greger et al., 2007; Lau and Zukin, 2007). Identification of the mechanisms governing composition and the number of active iGluRs at synapses is necessary to understand their role in synaptic activity and plasticity.

Different classes of cell adhesion molecules, such as cadherins (Boggon et al., 2002; Bamji, 2005), are involved in the regulation of synaptic trafficking and stabilization of iGluRs. N-Cadherin has a key role during dendrite arborization, axon guidance, and synaptogenesis (Benson and Tanaka, 1998; Yu and Malenka, 2003). Moreover, at synapses, $\mathrm{N}$-cadherin regulates synaptic adhesion, spine morphology, and activity-dependent plasticity (Togashi et al., 2002; Okamura et al., 2004; Xie et al., 2008; Mendez et al., 2010). Recent studies also showed that N-cadherin is mandatory for an increase in AMPA receptor content and function at synaptic sites (Liao et al., 1999; Okuda et al., 2007; Saglietti et al., 2007; Tai et al., 2008; Xie et al., 2008), which is associated

Received April 19, 2010; revised Sept. 2, 2010; accepted Oct. 4, 2010.

This work was supported by the European Union Seventh Framework Program (FP7 2007-2013) under Grant Agreement PIAP-GA-2008-217902 (M.D.L.) and by Fondazione CARIPL0 2008 (M.D.L.). C.S. was supported by Telethon-Italy Grant GGP09196, Fondazione CARIPLO Project 2009.264, and RSTL-CNR and Regione Lombardia Project SAL-50-16983 TERDISMENTAL. We thank Elisa Zianni for excellent technical assistance and Maria Passafaro for GFP-N-cadherin construct.

Correspondence should be addressed to Monica Di Luca, Department of Pharmacological Sciences, University of Milan, Via Balzaretti 9, 20133 Milan, Italy. E-mail: monica.diluca@unimi.it.

DOI:10.1523/JNEUROSCI.1984-10.2010

Copyright $\odot 2010$ the authors $\quad 0270-6474 / 10 / 3016343-13 \$ 15.00 / 0$ with spine enlargement (Kasai et al., 2003; Abe et al., 2004; Mendez et al., 2010). Thus, a temporal and functional relationship between N-cadherin, synaptic accumulation of AMPA receptors, and spine growth has been put forward (Zito et al., 2009).

Recent data showed that N-cadherin can be cleaved by ADAM10 (a disintegrin and metalloproteinase 10). Via its metalloproteinase domain, the enzyme is responsible for the initial step of N-cadherin processing (Reiss et al., 2005; Uemura et al., 2006), which, extracellularly, releases soluble active fragments and, intracellularly, generates a C-terminal fragment (CTF). CTF initiates signaling pathways through the cytoplasmic $\beta$-catenin pool (Fahrenholz and Postina, 2006; Reiss et al., 2006). Therefore, ADAM10-dependent cleavage of N-cadherin modulates cell-cell adhesion as well as signal transduction. ADAM10 shows postsynaptic localization (Janes et al., 2005; Marcello et al., 2007) permitted by association with synapse-associated protein-97 (SAP97), a member of the postsynaptic density (PSD)membrane-associated guanylate kinase (MAGUK) protein family. Perturbing ADAM10/SAP97 association impairs ADAM10 localization in the postsynaptic membrane and decreases ADAM10 activity toward synaptic substrates (Marcello et al., 2007).

What are the consequences of ADAM10 activity on N-cadherin at synaptic sites? And how may this processing contribute to spine remodeling and availability/stabilization of active iGluRs, which have been described as linked to $\mathrm{N}$-cadherin? To tackle this issue, we used the tissue inhibitor of metalloproteinase-1 (TIMP-1), an ADAM10 inhibitor, as well as a cell-permeable peptide (TATPro) able to interfere in vitro and in vivo with ADAM10 synaptic localization and activity. Inhibition of ADAM10 activity on $\mathrm{N}$-cadherin induced a significant increase in size of dendritic spines and a modification of the number and the currents of synaptic AMPA receptors. 
A

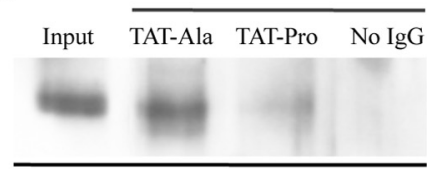

WB: SAP97
B

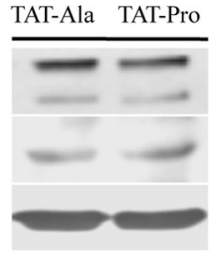

C TAT-Ala TAT-Pro

SAP97

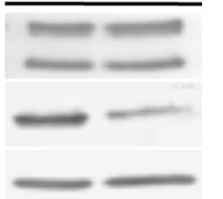

SAP97

ADAM10

ADAM10

Tubulin
Tubulin
D

TAT-Ala
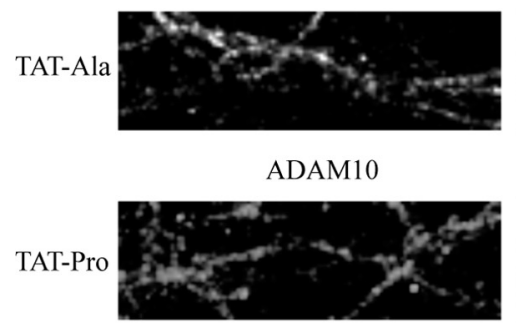

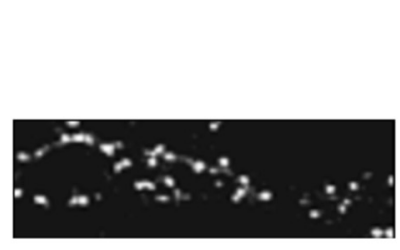

Shank

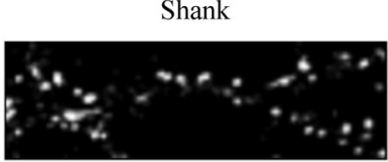

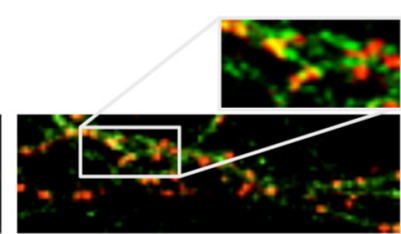

Merge

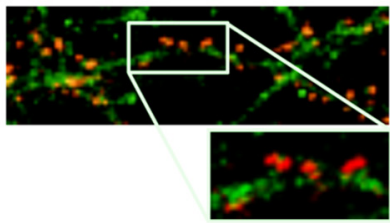

E

TAT-Ala TAT-Pro

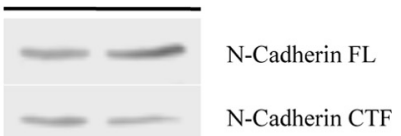

Tubulin

Figure 1. ADAM10/SAP97 interaction is required for ADAM10-mediated cleavage of synaptic N-cadherin. A, DIV10 primary hippocampal neurons were treated with TAT-Ala and TAT-Pro ADAM10 peptides (10 $\mu \mathrm{M}, 30 \mathrm{~min}$ ) and lysed. ADAM10 was immunoprecipitated (IP) from total homogenate with an antibody raised against its N-terminal domain, and Western blot (WB) analysis was performed with an antibody against SAP97. Treatment with TAT-Pro was able to reduce coimmunoprecipitation of SAP97 with ADAM10 $(p<0.005 ; n=3)$ compared with TAT-Ala. $\boldsymbol{B}$, Western blot analysis performed in total homogenate with antibodies against ADAM10 and SAP97: no change in ADAM10 ( $p=0.4423 ; n=7)$ and SAP97 $(p=0.7692 ; n=7)$ levels was observed. C, Western blot analysis performed in TIF with antibodies against ADAM10 and SAP97. TAT-Pro decreased ADAM10 ( $p<0.0005 ; n=7)$ but not of SAP97 $(p=0.8488 ; n=7)$ localization in the TIF. D, DIV10 primary hippocampal neurons were immunolabeled for ADAM10 (green) and Shank (red) as the postsynaptic marker. TAT-Pro ADAM10 peptide decreased ADAM10/Shank colocalization ( $p<0.05$; $n=10)$. $E$, Western blot analysis performed in TIF with an antibody against the C-terminal domain of N-cadherin. Treatment with TAT-Pro induced a statistically significant increase, when compared with TAT-Ala, in N-cadherin FL in the TIF ( $p<0.05 ; n=7)$, a concomitant decrease in CTF $(p<0.05 ; n=7)$, and a consequent increase in the FL/CTF ratio $(p<0.005 ; n=6)$.

\section{Materials and Methods}

Antibodies, reagents, and cell-permeable peptides. The following antibodies $(\mathrm{Ab})$ were used: monoclonal antibody $(\mathrm{mAb})$ anti-green fluorescent protein (GFP) and mAb anti-pan Shank (NeuroMab, University of California Davis, Davis, CA); mAb anti- $\alpha$-tubulin, anti-actin IgG fraction of antiserum (20-33 amino acids; Sigma), and polyclonal antibody (pAb) anti-ADAM10 $\mathrm{N}$ terminal (Abcam); mAb anti-NMDA NR1, pAb anti$\mathrm{NR} 2 \mathrm{~B}$, and mAb anti-NR2A (Zymed Laboratories); pAb anti-AMPA GluR1, mAb anti-GluR2, goat pAb anti-AKAP 150, and mAb anti-PYK$2 y 67$ (Millipore); mAb anti-SAP97 (Stressgen) and mAb C-32 anti-Ncadherin C-terminal domain (BD Biosciences); pAb anti-GFP and Alexa Fluor secondary Abs (Invitrogen); and peroxidase-conjugated secondary Abs (Pierce).

TIMP-1 was purchased from Calbiochem; (+)-bicuculline was purchased from Tocris Biosciences, and TTX was purchased from Ascent Scientific.

TAT-Pro ${ }^{709-729}$ ADAM10 inhibitory peptide (TAT-Pro) was obtained linking the 11 amino acid human immunodeficiency virus TAT transporter sequence (Aarts et al., 2002) to the 21 amino acid sequence corresponding to ADAM10 proline-rich domains $\left(\mathrm{NH}_{2}\right.$-YGRKKRRQRRR-PKLPPPKPLPGTLKRRRPPQP-COOH). TAT-Ala ${ }^{709-729}$ ADAM10 peptide (TAT-Ala), in which all proline residues were mutated into alanine $\left(\mathrm{NH}_{2}-\right.$ YGRKKRRQRRR-AKLAAAKALAGTLKRRRAAQA-COOH), was used as the control (Marcello et al., 2007). Non-cell-permeable peptide lacking TAT sequence mimics the 21 amino acid sequence of ADAM10 proline-rich domains (Pro ADAM10 peptide, $\mathrm{NH}_{2}$-PKLPPPKPLPGTLKRRRPPQP$\mathrm{COOH})$. As the negative control, a similar peptide in which all proline residues were mutated into alanine (Ala ADAM10 peptide, $\mathrm{NH}_{2}$-AKLAAAKALAGTLKRRRAAQA-COOH) was used. All peptides were synthesized by XigenPharma (Lausanne, Switzerland).

GFP-N-cadherin plasmid was provided by M. Passafaro (University of Milan, Milan, Italy); GFP-N-cadherin cleavage defective (GFP-Ncadherin GD) was created by site-directed mutagenesis substitution of both $\mathrm{R}^{714} \rightarrow \mathrm{G}^{714}$ and $\mathrm{I}^{715} \rightarrow \mathrm{D}^{715}$ as described previously (Uemura et al., 2006).

Immunoprecipitation. Fifty micrograms of homogenate from cultured hippocampal neurons, acute hippocampal slices, or mouse brain treated with both TAT-Pro and TAT-Ala peptides were incubated in RIA buffer (200 mM NaCl, 10 mm EDTA, $10 \mathrm{~mm} \mathrm{Na}_{2} \mathrm{HPO}_{4}, 0.5 \% \mathrm{NP}-40$, and $0.03 \%$ SDS) in a final volume of $200 \mu \mathrm{l}$ with antibody against ADAM10 $\mathrm{N}$-terminal domain overnight at $4^{\circ} \mathrm{C}$. Protein A-Sepharose beads (SigmaAldrich) were added, and incubation was continued for $2 \mathrm{~h}$, at room temperature, with shaking. Beads were collected by centrifugation at $1000 \times g$ for $5 \mathrm{~min}$ and washed three times with RIA buffer before adding sample buffer for SDS-PAGE and boiling for $5 \mathrm{~min}$. Beads were collected by centrifugation, and all supernatants were applied onto $6 \%$ SDS-PAGE.

Animal treatment. Two-week-old mice (Charles River) received a single intraperitoneal injection of either TAT-Pro $(3 \mathrm{nmol} / \mathrm{g})$ or TAT-Ala peptide $(3 \mathrm{nmol} / \mathrm{g})$ diluted in sterile saline solution (Marcello et al., 2007). Animals were killed after $24 \mathrm{~h}$, and the brains were rapidly removed. All experimental procedures were performed with care to minimize discomfort and pain to treated animals, in accordance with the guidelines of the European Communities Council (Directive 86/609/ EEC of November 24, 1986). One hemisphere of each animal was homogenized in $0.32 \mathrm{M}$ ice-cold sucrose containing $1 \mathrm{~mm}$ HEPES, $1 \mathrm{~mm}$ $\mathrm{MgCl}_{2}, 1 \mathrm{~mm}$ EDTA, 1 mм $\mathrm{NaHCO}_{3}$, and $0.1 \mathrm{~mm}$ PMSF, pH 7.4, in the presence of a complete set of proteases inhibitors (Complete; GE Healthcare) and phosphatase inhibitors (Sigma-Aldrich). The homogenized tissue was centrifuged at $1000 \times g$ for $10 \mathrm{~min}$. The resulting supernatant (S1) was centrifuged at $13,000 \times g$ for 15 min to obtain a crude membrane fraction (P2 fraction). The pellet was resuspended in $1 \mathrm{~mm}$ HEPES plus Complete in a glass-glass homogenizer and centrifuged at $100,000 \times \mathrm{g}$ for $1 \mathrm{~h}$. The pellet (P3) was resuspended in buffer containing $75 \mathrm{~mm} \mathrm{KCl}$ and $1 \%$ Triton X-100 and centrifuged at 100,000 $\times g$ for $1 \mathrm{~h}$. The final pellet [P4, Triton-insoluble postsynaptic fraction (TIF)] was homogenized in $20 \mathrm{~mm}$ HEPES plus Complete in a glass-glass homogenizer.

Acute hippocampal slices, neuronal culture preparation, and transfection. Acute hippocampal slices were prepared as described previously (Gardoni et al., 2001, 2003). Slices were quickly frozen at $-80^{\circ} \mathrm{C}$ after pharmacological treatments. Hippocampal neuronal primary cultures were prepared from embryonic day 18-19 rat hippocampi as described 
A

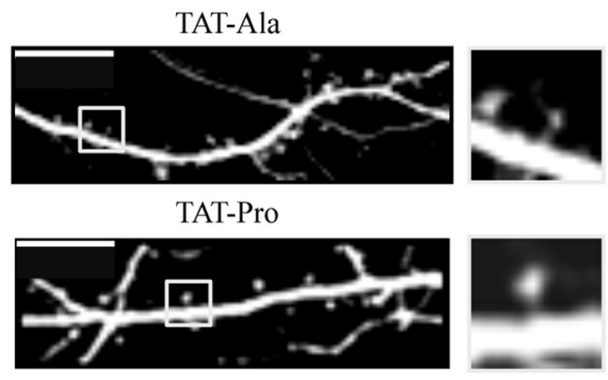

D
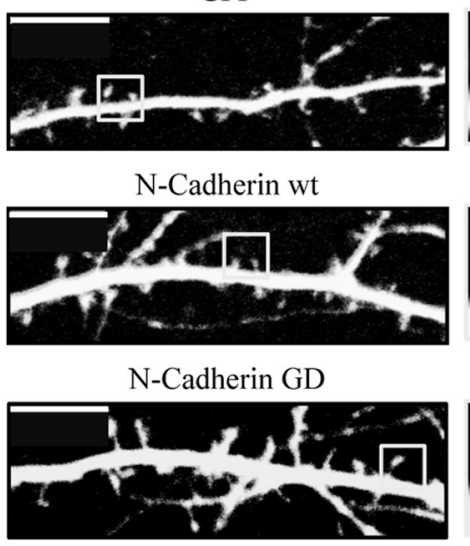

B
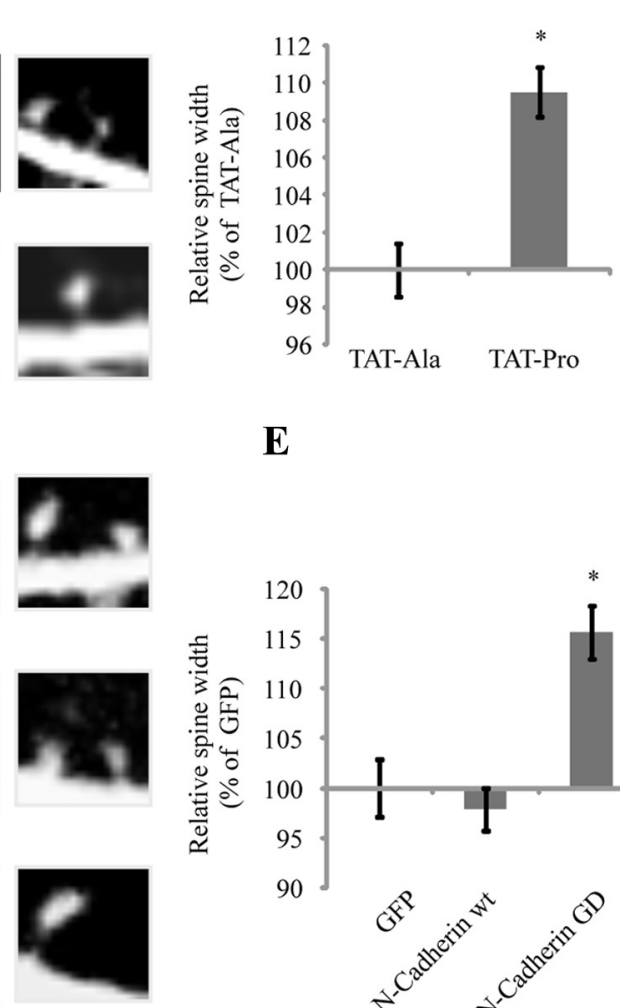

$\mathbf{E}$

TAT-Ala TAT-Pro

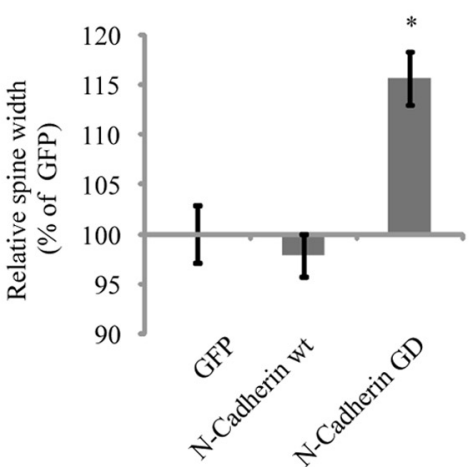

$\mathbf{C}$

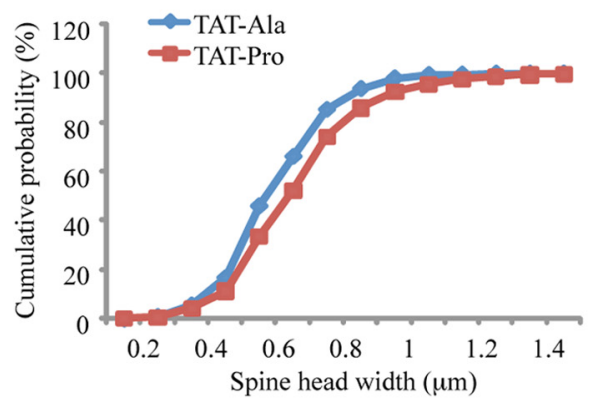

F

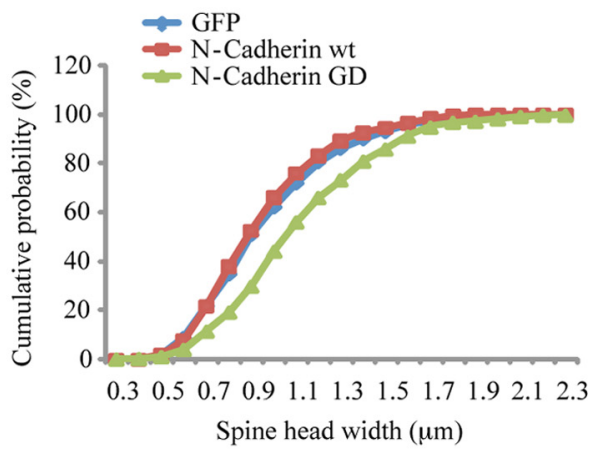

Figure 2. ADAM10 localization and activity influence spine morphology. A, Primary hippocampal neurons were transfected with GFP construct at DIV7 and treated at DIV10 with TAT-Ala and TAT-Pro ADAM10 peptides (10 $\mu \mathrm{m}, 30 \mathrm{~min}$ ). Cells were fixed and immunolabeled for GFP. Representative images show dendrites from neurons treated with TAT-Ala and TAT-Pro ADAM10 peptides. Scale bar, $10 \mu \mathrm{m} . \boldsymbol{B}$, Diagram showing relative average spine head width ( ${ }^{*} p<0.0005$, TAT-Pro vs TAT-Ala; $n>2600$ spines from 14 different neurons for each group). $C$, Cumulative frequency plots from neurons treated with TAT-Ala and TAT-Pro. D, Neurons cotransfected at DIV7 with DS-RED and GFP, GFP-N-cadherin Wt, or GFP-N-cadherin GD. Cells were fixed, and images were taken for DS-RED. Scale bar, $10 \mu \mathrm{m}$. $E$, Diagram showing relative average spine head width ( $p<0.0001$ among groups, $p<0.001 \mathrm{GD}$ vs GFP, $p<0.0001 \mathrm{GD}$ vs Wt; $n>500$ spines from 10 different neurons for each group). $\boldsymbol{F}$, Cumulative frequency plots from neurons transfected with GFP, GFP-N-cadherin Wt, or GFP-N-cadherin GD.

previously (Piccoli et al., 2007). Neurons were transfected at $7 \mathrm{~d}$ in vitro (DIV7) with GFP, DS-RED, GFP-N-cadherin wild type (Wt), and GFP$\mathrm{N}$-cadherin GD constructs using the calcium-phosphate method.

Subcellular fractionation and surface expression assay. TIFs were isolated from neurons as described previously (Gardoni et al., 2009). Crosslinking experiments by means of bis(sulfosuccinimidyl) suberate $\left(\mathrm{Bs}^{3}\right)$ (Pierce) were performed as described previously (Hall and Soderling, 1997; Mauceri et al., 2004).

Immunocytochemistry. Transfected neurons treated with both TATPro and TAT-Ala peptide (10 $\mu \mathrm{M}, 30 \mathrm{~min}$ ) were fixed for $30 \mathrm{~min}$ in $4 \%$ paraformaldehyde at room temperature and immunostained for GFP; primary and secondary antibodies were applied in GDB buffer $(30 \mathrm{~mm}$ phosphate buffer, $\mathrm{pH} 7.4$, containing $0.2 \%$ gelatin, $0.5 \%$ Triton $\mathrm{X}-100$, and $0.8 \mathrm{M} \mathrm{NaCl}$ ) (Sala et al., 2001). Cells were chosen randomly for quantification from four different coverslips (two independent experiments), and 14 neurons for each treatment were analyzed. Fluorescence images were acquired by using the confocal LSM510 Meta system (Zeiss) with a $63 \times$ objective and a sequential acquisition setting at $1024 \times 1024$ pixel resolution; for each image, three to four $1 \mu \mathrm{m}$ sections were acquired, and $z$-projection was obtained.

For colocalization studies, hippocampal neurons treated with TATPro and TAT-Ala peptide (10 $\mu \mathrm{M}, 30 \mathrm{~min})$, or with TIMP-1 (15 nM, 30 $\mathrm{min}$ ), were fixed for $30 \mathrm{~min}$ in $4 \%$ paraformaldehyde at room temperature and immunostained for GluR1, ADAM10, and Pan-Shank as the postsynaptic marker; primary and secondary antibodies were applied in GDB buffer (30 mm phosphate buffer, $\mathrm{pH} 7.4$, containing $0.2 \%$ gelatin, $0.5 \%$ Triton X-100, and $0.8 \mathrm{M} \mathrm{NaCl}$ ) (Sala et al., 2001). Cells were chosen randomly for quantification from four different coverslips (two independent experiments), and 10 neurons for each treatment were analyzed. Fluorescence images were acquired by using the confocal LSM510 Meta system (Zeiss) with a $63 \times$ objective and a sequential acquisition setting at $1024 \times 1024$ pixel resolution; colocalization analysis was performed with the confocal software (Zeiss).

Morphological analysis of in vivo experiments was conducted by using a diolistic gene gun system to propel DiI-coated particles into sections of fixed brain tissue. Images of DiI-labeled structures in hippocampal CA1 stratum radiatum were acquired using the LSM510 confocal microscope (Zeiss). At least $10 z$-stack images were acquired for each animal and for each area of interest. Morphometric analysis of dendritic spines was performed with MetaMorph software.

Live imaging. Time-lapse imaging occurred in an environmentally controlled chamber with $5 \%$ carbon dioxide at $37^{\circ} \mathrm{C}$, and images were obtained using the confocal LSM510 Meta system (Zeiss) with a $63 \times$ objective and zoom function set on 4 ; for each image, three to four $1 \mu \mathrm{m}$ sections were acquired, and $z$-projection was obtained. Images were acquired every $3 \mathrm{~min}$ for $30 \mathrm{~min}$ of nontreated cells used as the control, after injection of TAT-Pro or TAT-Ala peptide images were acquired every $3 \mathrm{~min}$ for $30 \mathrm{~min}$. Head width of all spines was measured at each time point.

Electrophysiological recordings from CA1 pyramidal cells. Experiments were performed on parasaggital hippocampal slices from 14- to 16-d-old C57BL/6 mice by using standard techniques. Whole-cell voltage-clamp recordings (3.5-4.5 $\mathrm{M} \Omega$ electrodes) were made at $30-32^{\circ} \mathrm{C}$ from pyramidal cells of the CA1 field visualized by infrared videomicroscopy. Slices were perfused with extracellular solution composed of $125 \mathrm{~mm} \mathrm{NaCl}, 2.5$ mм KCl, $1.25 \mathrm{~mm} \mathrm{NaH}_{2} \mathrm{PO}_{4}, 26 \mathrm{~mm} \mathrm{NaHCO}, 2.3 \mathrm{~mm} \mathrm{CaCl}_{2}, 1.3 \mathrm{~mm}$ $\mathrm{MgCl}_{2}$, and $25 \mathrm{~mm}$ glucose saturated with $95 \% \mathrm{O}_{2} / 5 \% \mathrm{CO}_{2}$. Bicuculline $(10 \mu \mathrm{M})$ was added to the bath to block $\mathrm{GABA}_{\mathrm{A}}$ receptors. The intracellular solution was composed of $140 \mathrm{~mm}$ cesium methanesulfonate, $2 \mathrm{mM}$ $\mathrm{MgCl}_{2}, 4 \mathrm{~mm} \mathrm{NaCl}, 5 \mathrm{~mm}$ phospho-creatine, $2 \mathrm{~mm} \mathrm{Na}_{2} \mathrm{ATP}, 10 \mathrm{~mm}$ EGTA, $10 \mathrm{~mm}$ HEPES, and $0.33 \mathrm{~mm}$ GTP, pH 7.3. In experiments in 

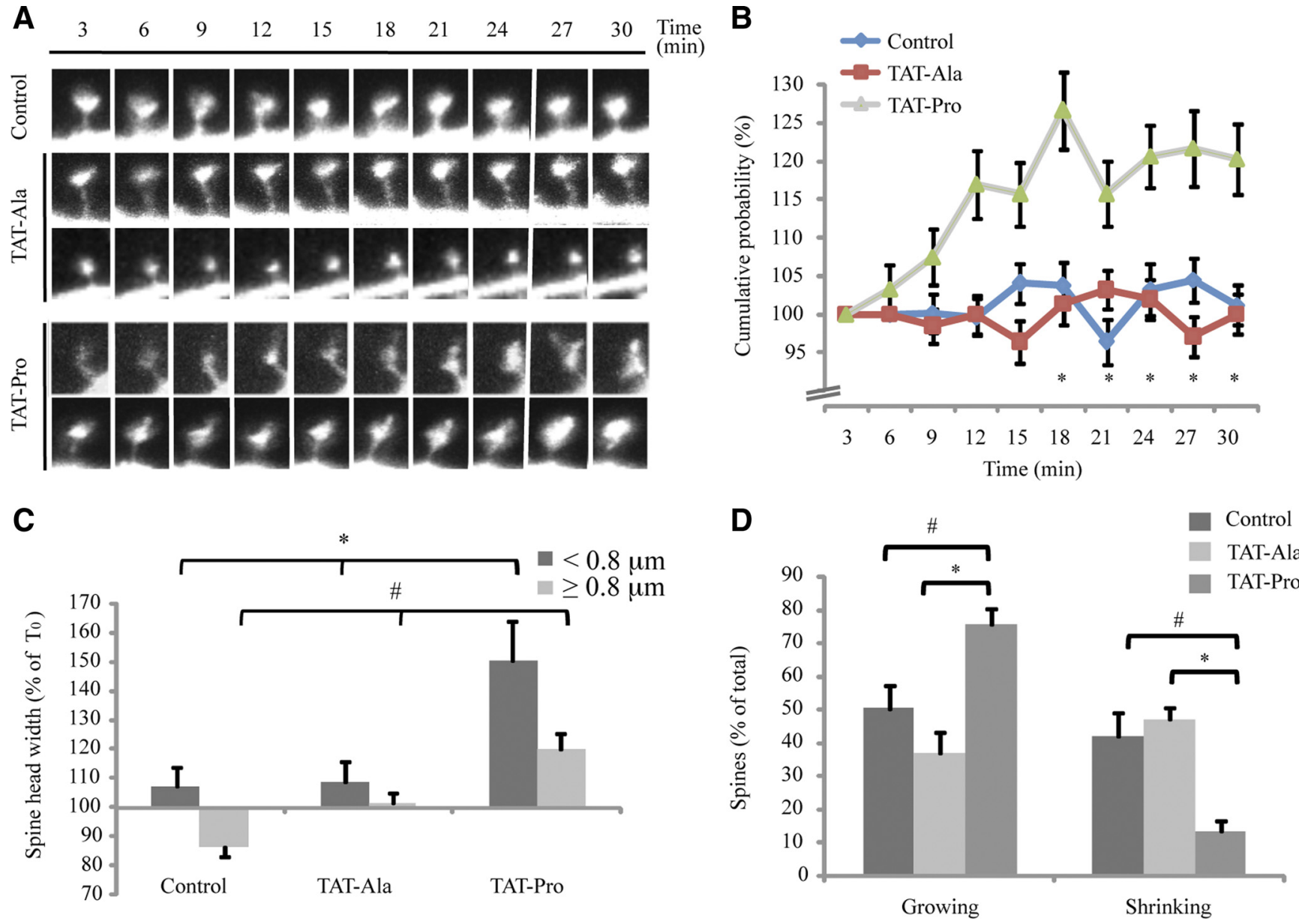

Figure 3. Timing of ADAM10-dependent effect on spine morphology. $A$, Representative time-lapse imaging of hippocampal neurons transfected with GFP (DIV7) and incubated (DIV10) with TAT-Ala or TAT-Pro ADAM10 peptides $(10 \mu \mathrm{m})$. B, Spine head width was measured at all set times and expressed as a percentage of initial values; TAT-Pro increased spine head width compared with control and TAT-Ala-treated neurons ( $p<0.001$ among groups, $p<0.05$ TAT-Pro vs control, $p<0.05$ TAT-Pro vs TAT-Ala; $n>60$ spines from 4 independent experiments for each group). $C$, Dendritic spines have been divided into two groups depending on their head width (cutoff, $0.8 \mu \mathrm{m}$ ). For each group of spines, the average head width at end point ( $30 \mathrm{~min}$ ) has been calculated and expressed as a percentage of the initial value. TAT-Pro increased average head width in both populations of dendritic spines when compared with control and TAT-Ala-treated neurons (width $<0.8$ $\mu \mathrm{m}: p<0.01$ among groups, ${ }^{*} p<0.01$ TAT-Pro vs control, ${ }^{*} p<0.05$ TAT-Pro vs TAT-Ala; width $\geq 0.8 \mu \mathrm{m}: p<0.0001$ among groups, ${ }^{\#} p<0.0001$ TAT-Pro vs control, ${ }^{\#} p<0.005$ TAT-Pro vs TAT-Ala; $n>60$ spines from 4 independent experiments for each group). $D$, Diagram showing statistical analysis of growing and shrinking spines after a 30 min treatment with TAT-Pro or TAT-Ala peptides as a percentage of the total number of spines. TAT-Pro ADAM10 induced a statistically significant increase in the percentage of growing spines $\left(p<0.01\right.$ among groups, ${ }^{\#} p<0.05$ TAT-Pro vs control, ${ }^{*} p<0.01$ TAT-Pro vs TAT-Ala; $n>60$ spines from 4 independent experiments for each group) and a statistically significant decrease in shrinking ones ( $p<0.01$ among groups, ${ }^{\#} p<$ 0.05 TAT-Pro vs control, ${ }^{*} p<0.01$ TAT-Pro vs TAT-Ala; $n>60$ spines from 4 independent experiments for each groups).

which the rectification indices (RIs) were measured, $1 \mathrm{~mm}$ QX314 and 0.1 mM spermine were also added to the intracellular solution. Evoked postsynaptic responses were induced in CA1 pyramidal cells by stimulating Schaffer collaterals in stratum radiatum $(0.1 \mathrm{~Hz})$ with a monopolar glass pipette. In experiments shown in Figure 5, nonpermeable ADAM10 Pro and Ala peptides were delivered postsynaptically through the recording pipette. We waited 15 min after establishing the whole-cell configuration before starting the recording to ensure adequate diffusion of reagents into dendritic compartments. RIs were calculated by plotting the magnitude of the average EPSC at $-60,0$, and $+40 \mathrm{mV}$ and taking the ratio of the slope of the lines connecting values at $0-40$ and at -60 to 0 $\mathrm{mV}$. This calculation, with respect to the simple ratio of EPSC amplitude at $+40 /-60 \mathrm{mV}$, takes into account the variable AMPA receptor reversal potential of each recording (Adesnik and Nicoll, 2007). In experiments shown in Figure 8, mice were given intraperitoneal injections of ADAM10 TAT-Ala or TAT-Pro peptides ( $3 \mathrm{nmol} / \mathrm{g}$ ) $24 \mathrm{~h}$ before slice preparation. AMPA receptor EPSCs were recorded at $-80 \mathrm{mV}$. NMDA receptor EPSCs were recorded at $+40 \mathrm{mV}$ and measured $40 \mathrm{~ms}$ after the initiation of the EPSC, a time point at which AMPA receptor-mediated currents are absent or minimal. Small hyperpolarizing voltage steps were given before each afferent stimulus allowing on-line monitoring of input and series. Values are expressed as mean \pm SEM.
Quantification and statistical analysis. Quantification of Western blot analysis was performed by means of computer-assisted imaging (ImageJ) after normalization on actin or tubulin levels, and values were expressed as mean \pm SEM. Colocalization analysis was performed using AIM 4.2 software (Zeiss).

Statistical evaluations were performed by using the unpaired Student's $t$ test or, when appropriate, by using one-way ANOVA (Fig. 2D-F, 3, 4G, and $9 E$ ), followed by Bonferroni's post hoc test.

For electrophysiological experiments shown in Figure 5, we used twoway ANOVA among groups perfused with Pro and Ala peptide and the unpaired Student's $t$ test for the last 2 min of recording. For electrophysiological experiments shown in Figure 8, the unpaired Student's $t$ test was used for data comparison.

\section{Results}

ADAM10/SAP97 interaction is required for ADAM10-mediated cleavage of synaptic $\mathrm{N}$-cadherin

First, we asked whether constitutive ADAM10-mediated cleavage of $\mathrm{N}$-cadherin occurs at synapses of primary hippocampal neurons during neuronal development (DIV10). To this, we interfered with ADAM10 activity by means of a previously char- 
A

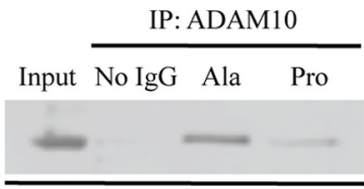

WB: SAP97

B

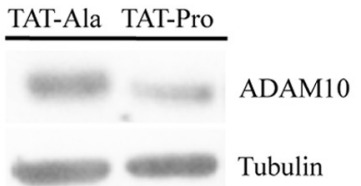

F

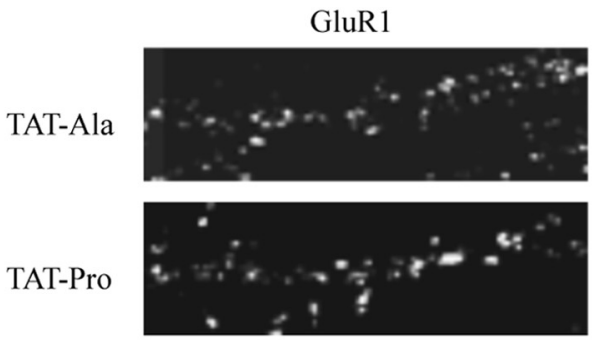

G

GFP

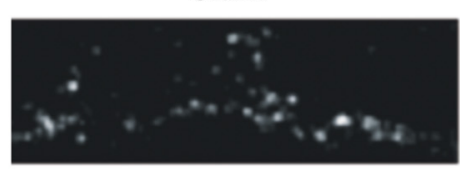

$\mathrm{N}$-Cadherin wt

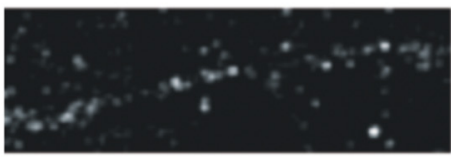

N-Cadherin GD

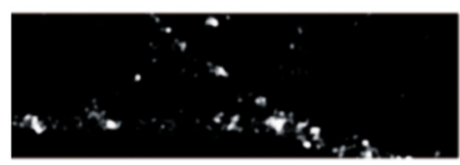

D

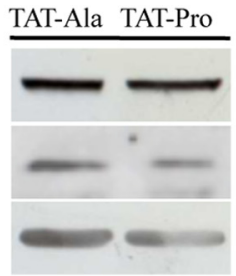

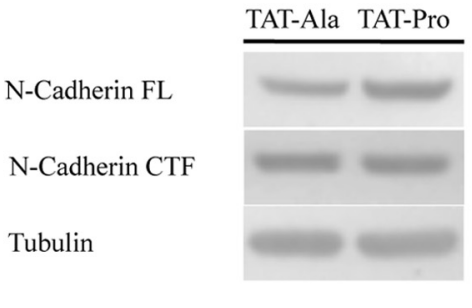

Shank
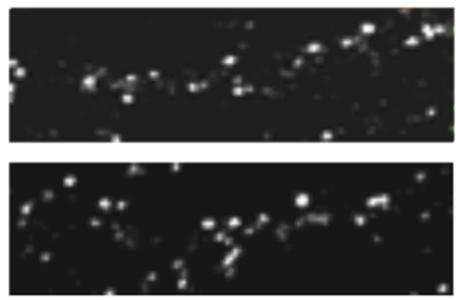

Shank
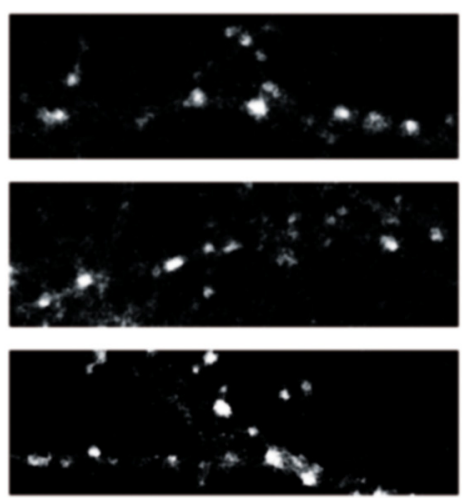

$\mathbf{E}$
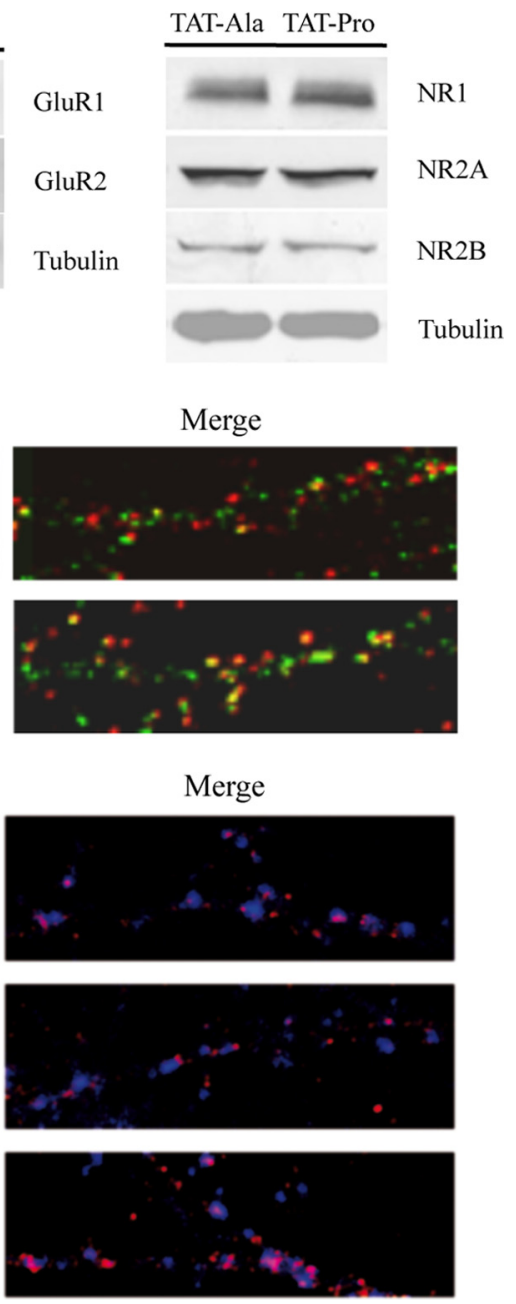

Figure 4. Role of ADAM10 synaptic localization on AMPA receptor composition. A, Acute hippocampal slices were treated with TAT-Pro or TAT-Ala ADAM10 peptides (10 $\mu$ M, 30 min). Total homogenate was immunoprecipitated with an antibody against ADAM10, and Western blot analysis for SAP97 was performed: treatment with TAT-Pro inhibitory peptide reduced SAP97 coimmunoprecipitation with ADAM10 ( $p<0.005 ; n=3)$. B. Treatment with TAT-Pro induced a statistically significant decrease in ADAM10 localization in the TIF $(p<0.05 ; n=5)$. C, TAT-Pro inhibitory peptide induced an increase in the FL/CTF N-cadherin ratio $(p<0.05 ; n=3)$ compared with TAT-Ala. $\boldsymbol{D}$, Western blot analysis performed in the TIF shows a statistically significant increase in GluR1 $(p<0.0001 ; n=6)$, but no modification of the GluR2 $(p=0.9399 ; n=6)$ subunit of AMPA receptor. $E$, Western blot analysis shows no modification of NMDA receptor subunits in TIF after treatment with TAT-Pro (NR1, $p=0.9728 ; \mathrm{NR2A}, p=0.4375 ; \mathrm{NR2B}, p=0.9839 ; n=4)$. $F$, Immunolabeling of primary hippocampal neurons for GluR1 (green) and Shank (red); TAT-Pro increased GluR1/Shank colocalization ( $p<0.0005, n=10)$. G, Immunolabeling of primary hippocampal neurons for GluR1 (red) and Shank (blue); transfection with N-cadherin GD but not with $\mathrm{N}$-cadherin Wt increased GluR1/Shank colocalization ( $p<0.0002$ among groups, $p<0.002$ GD vs GFP, $p<0.0002 \mathrm{GD} \mathrm{vs} \mathrm{Wt;} n>21$ from 3 independent experiments for each group).

acterized cell-permeable peptide (Marcello et al., 2007), in which the TAT cell-permeable domain is fused to the C-terminal proline-rich domains of ADAM10 (TAT-Pro) (supplemental Fig. $1 A$, available at www.jneurosci.org as supplemental material). TAT-Pro peptide competes with endogenous ADAM10 for the binding to the SH3 domain of SAP97 and disrupts the endogenous ADAM10/SAP97 complex (Marcello et al., 2007). The ADAM10/SAP97 complex is responsible for the enzyme localization and activity at synaptic membranes, and treatment with the peptide is able to reduce both ADAM10 localization and activity at synapses (Marcello et al., 2007). As the control, an analogous inactive peptide in which all proline residues were substituted by alanines was used (TAT-Ala). Coimmunoprecipitation experiments were performed after treatment of primary hippocampal neurons (DIV10) with TAT-Pro or TAT-Ala peptides (10 $\mu \mathrm{M}, 30$ min) (Fig. $1 A$ ). A reduction in ADAM10/SAP97 coimmunoprecipitation was observed in neurons treated with TAT-Pro inhib- itory peptide, indicating a decreased presence of the ADAM10/ SAP97 complex (Fig. 1 ) (TAT-Pro vs TAT-Ala, $-29.2 \pm 1.1 \%$; $p<0.005)$. Under these experimental conditions, we found no modifications of SAP97 and ADAM10 levels in total homogenate, suggesting that the observed uncoupling can be ascribed to a decreased association rather than to changes in levels of these proteins (Fig. $1 B$ ). A significant reduction in ADAM10 in TIF, enriched in proteins of the postsynaptic density (Gardoni et al., 2009) was observed (Fig. 1C; TAT-Pro vs TAT-Ala, $-39.6 \pm$ $5.8 \% ; p<0.0005)$, indicating a decreased ADAM10 synaptic localization. Biochemical data were also confirmed by immunocytochemical labeling of ADAM10 in hippocampal neurons (DIV10). As shown in Figure $1 D$, treatment with Tat-Pro led to a reduction in ADAM10 punctate staining in Shank-positive clusters (TAT-Pro vs TAT-Ala, $-45.3 \pm 8.3 \%$; $p<0.05$ ).

To check TAT-Pro specificity to disrupt the ADAM10/SAP97 complex without influencing other protein-protein interactions, 
A
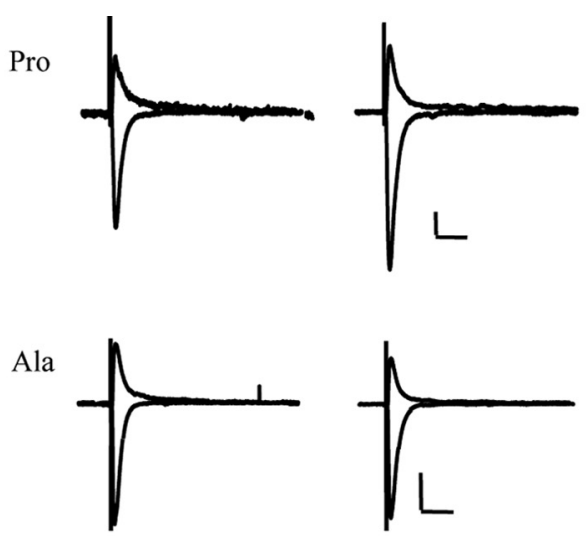

B

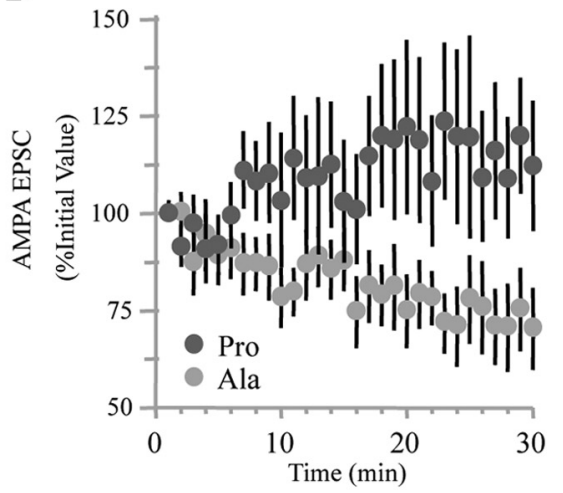

C

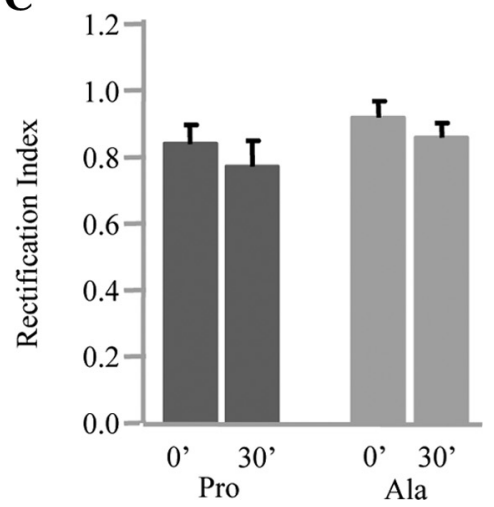

Figure 5. Role of ADAM10 synaptic localization on AMPA receptor activity. A, Averaged AMPA receptor EPSCs (6 responses) recorded at -60 and $+40 \mathrm{mV}$ from CA1 pyramidal cells with an intracellular solution containing spermidine $(0.1 \mathrm{mM})$ and Pro or Ala ADAM10 peptides. Recordings were started after $15 \mathrm{~min}$ after entering in whole-cell configuration to ensure an adequate diffusion of reagents into dendritic compartments, and AMPA responses were pharmacologically isolated with bicuculline (10 $\mu \mathrm{M})$ and D-AP-5 ( $50 \mu \mathrm{M})$. Calibration: $50 \mathrm{pA}, 50 \mathrm{~ms}$. The amplitude of EPSCs was measured for the next $30 \mathrm{~min} . \boldsymbol{B}$, Summary of the changes in the amplitude of the AMPA receptor EPSCs in cells intracellularly perfused with Pro or Ala ADAM10 peptides ( $p<0.0001$ with two-way ANOVA among groups perfused with Pro and Ala peptide and $p<0.005$ by unpaired Student's $t$ test for the last 2 min of recording for Pro and Ala peptide; $n=10-11)$. Responses were normalized with respect to the first minute of recording. C, Summary of RIs of AMPA receptor EPSCs in cells intracellularly perfused with Pro or Ala ADAM10 peptides at 0 and 30 min (Pro peptide, $p=0.4$; Ala peptide, $p=0.37 ; n=6-8$ ).

we tested SAP97 SH3 domain capability to bind to other partners in the presence of TAT-Pro. No alterations were found in the association between Akap150 and SAP97 (supplemental Fig. 1 B, available at www.jneurosci.org as supplemental material). TATPro did not interfere with the binding of other synaptic $\mathrm{SH} 3$ to Pro-rich domains, as assessed by coimmunoprecipitation of PYK2 and PSD-95 (supplemental Fig. 1C, available at www.jneurosci.org as supplemental material). On the whole, these data confirm that TAT-Pro is specific for its capability to interfere with the ADAM10/ SAP97 complex (Marcello et al., 2007).

We then investigated whether the observed decrease in ADAM10 synaptic localization after TAT-Pro treatment was associated with inhibition of ADAM10 activity toward N-cadherin. By using an antibody raised against the $\mathrm{N}$-cadherin $\mathrm{C}$-terminal intracellular domain (C-32), we detected two main immunoreactive bands corresponding to the full-length (FL) N-cadherin and to its CTF, which has previously been described as deriving from ADAM10-mediated cleavage (Reiss et al., 2005; Uemura et al., 2006). After treatment with TAT-Pro, an increase in N-cadherin FL (Fig. $1 E$ ) (TAT-Pro vs TAT-Ala, $+42.6 \pm 12.7 \%$; $p<0.05$ ), a concomitant decrease in $\mathrm{N}$-cadherin CTF (Fig. $1 E$ ) (TAT-Pro vs TAT-Ala, $-32.5 \pm 8.6 \% ; p<0.05)$, and a consequent increase in the FL/CTF ratio (Fig. $1 E$ ) (TAT-Pro vs TAT-Ala, $+90.1 \pm$ $10.7 \%$; $p<0.005)$ were observed, compared to TAT-Ala.

Thus, interfering with ADAM10 localization and activity at the postsynaptic membrane by means of TAT-Pro, we demonstrated that constitutive ADAM10-mediated cleavage of $\mathrm{N}$-cadherin indeed occurs at synapses of primary hippocampal neurons during development.

\section{ADAM10 localization and activity at synapse influence spine morphology}

Since N-cadherin signaling leads to modulation of spine morphology and synapse stability (Abe et al., 2004; Xie et al., 2008; Mendez et al., 2010), we asked whether the increased synaptic accumulation of N-cadherin FL, as obtained by TAT-Pro treatment, could be paralleled by modifications of spine morphology.

DIV7 GFP-transfected hippocampal neurons were incubated, at DIV10, with TAT-Pro or TAT-Ala $(10 \mu \mathrm{M}, 30 \mathrm{~min})$. After treatment, cells were fixed and immunolabeled with an anti-GFP antibody (Fig. 2A), and average spine head width and number of dendritic spines were evaluated. Statistical analysis revealed a significant increase in spine head width (Fig. $2 B)(p<0.0005)$ after treatment with TAT-Pro, and cumulative frequency plots of spine head width confirmed a significant shift toward bigger spine size (Fig. 2C). No changes in the mean spine density were found (TAT-Ala, $3.75 \pm 0.29$ spines $/ 10 \mu \mathrm{m}$; TAT-Pro, $4.10 \pm$ 0.18 spines $/ 10 \mu \mathrm{m} ; p=0.1853$ ).

To confirm that the effects of ADAM10 inhibition on spine size are dependent on $\mathrm{N}$-cadherin cleavage, we repeated morphological analysis in neurons after transfection with GFP, with GFP$\mathrm{N}$-cadherin $\mathrm{Wt}$, or with the cleavage-defective GFP-N-Cadherin GD construct, mutated in the ADAM10 cleavage site (Uemura et al., 2006). Both N-cadherin constructs showed the same expression pattern at the postsynaptic site as demonstrated by colocalization analysis with Shank-positive clusters (data not shown).

Statistical analysis revealed a significant increase in spine head width in GFP-N-cadherin GD but not in GFP N-cadherin Wt transfected neurons (Fig. $2 D-F)(p<0.0001$ among groups, $p<$ $0.001 \mathrm{GD}$ vs GFP, $p<0.0001 \mathrm{GD}$ vs $\mathrm{Wt}$ ), thus confirming that blocking ADAM10-dependent cleavage of $\mathrm{N}$-cadherin leads per se to an increase in spine size.

After confirming that the increase in spine width is attributable to the blocking of ADAM10 cleavage of N-cadherin, we investigated the onset of the effect by time-lapse imaging. In basal conditions, no significant modification of average spine head width was observed over $30 \mathrm{~min}$ (Fig. $3 \mathrm{~A}, B$ ). Similarly, treatment with TAT-Ala did not induce any modification of spine size. An increase in spine head width (Fig. $3 A, B$ ), which became statistically significant $18 \mathrm{~min}$ after treatment, was observed in TAT-Pro-treated neurons if compared with control and TAT-Ala-treated ones (Fig. $3 B$ ) ( $p<0.001$ among groups, $p<0.05$ TAT-Pro vs control, $p<$ 0.05 TAT-Pro vs TAT-Ala).

Is this morphological effect of TAT-Pro treatment dependent on spine size? Evaluation of spine head width in DIV10 primary hippocampal neurons revealed that $0.8 \mu \mathrm{m}$ represents a cutoff value that allowed to split spines into two equal populations (supplemental Fig. 2, available at www.jneurosci.org as supplemental material). As shown in Figure 3C, even if the absolute differences 
A

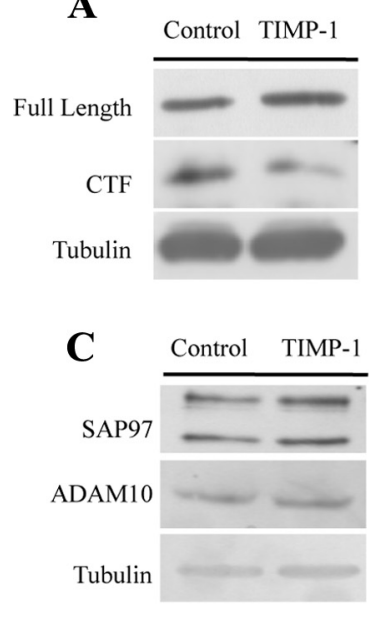

B

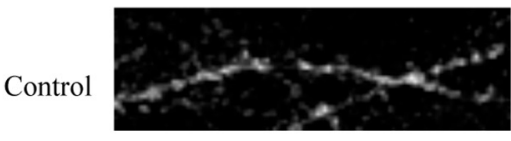

ADAM10

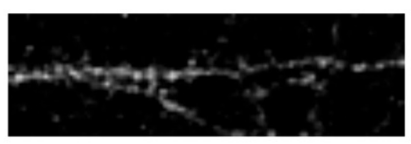

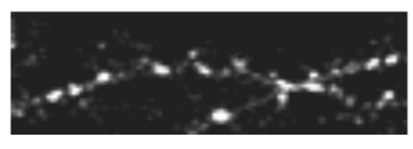

Shank

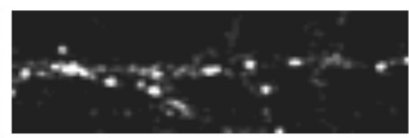

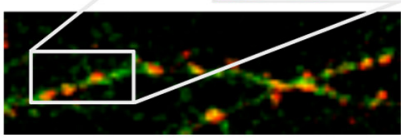

Merge

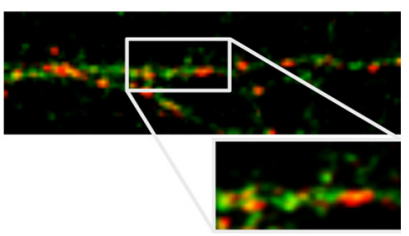

D

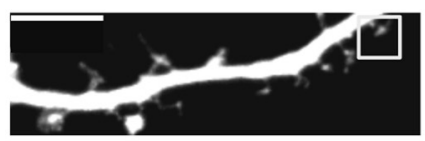

TIMP-1

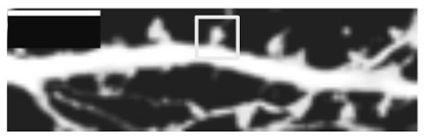

E

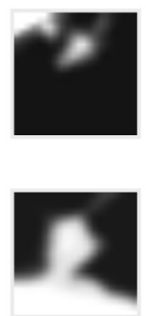

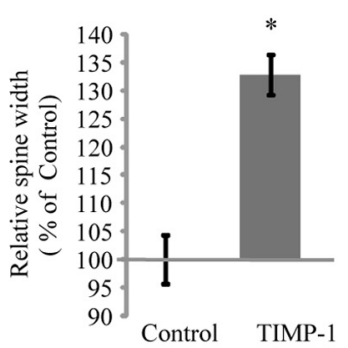

F

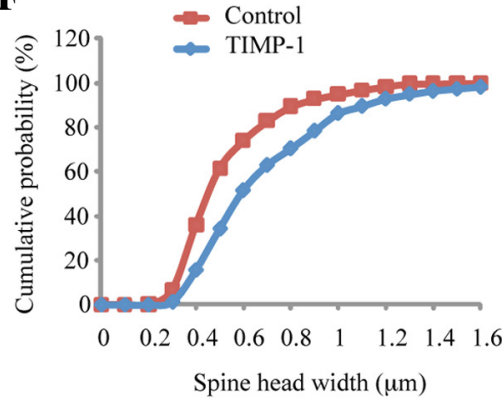

G

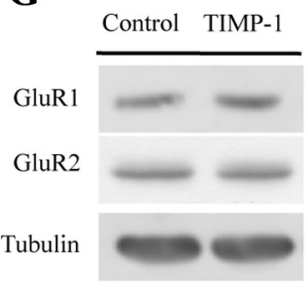

H

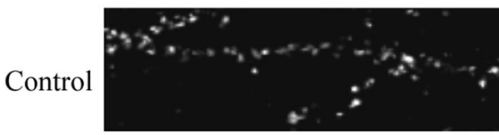

TIMP-1
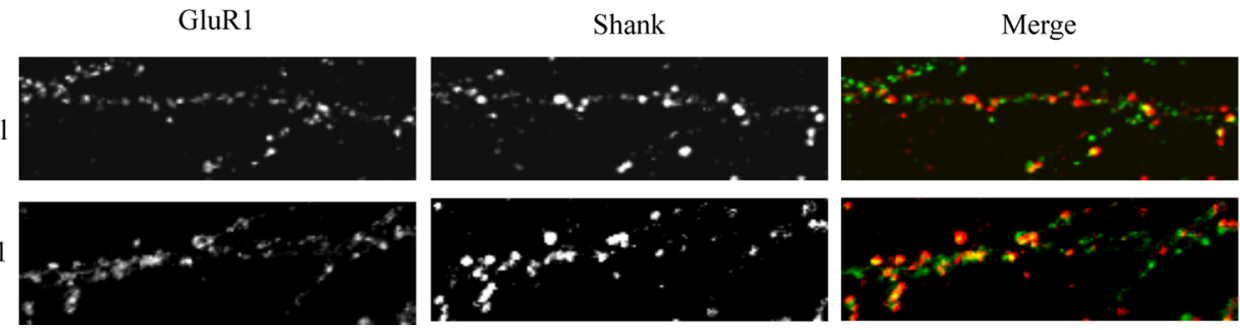

Figure 6. Inhibition of ADAM10 activity by TIMP-1 decreases ADAM10-mediated N-cadherin processing and induces a modification of AMPA receptors at synapses. $\boldsymbol{A}$, TIMP-1 (15 nM, 30 min) induced a statistically significant increase in N-cadherin FL in the TIF $(p<0.05 ; n=4)$, a concomitant decrease in CTF $(p<0.01 ; n=4)$, and a consequent increase in the FL/CTF ratio ( $p<0.005$; $n=4)$. $B$, DIV10 primary hippocampal neurons were immunolabeled for ADAM10 (green) and Shank (red). No modification of GluR1/Shank colocalization was observed after treatment with TIMP-1 $(p=0.3637 ; n=10)$. C, Western blot analysis performed on TIF with antibodies against ADAM10 and SAP97: no change in ADAM10 $(p=0.2240, n=6)$ and SAP97 $(p=0.9118, n=5)$ levels was observed. D, Primary hippocampal neurons were transfected with enhanced GFP construct (DIV7) and treated or not at DIV10 with TIMP-1 (15 nM, 30 min). Representative images show dendrites from control and TIMP-1-treated neurons. Scale bar, $10 \mu \mathrm{m}$. $E$, Diagram showing relative average spine head width $\left({ }^{*} p<0.0001\right.$, TIMP-1 vs control; $n>500$ spines from 9 different neurons for each condition). $\boldsymbol{F}$, Cumulative frequency plots from control and TIMP-1-treated neurons. $\boldsymbol{G}$, Western blot analysis performed in the TIF showed a statistically significant increase in GluR1 ( $p<$ $0.0001 ; n=5$ ) but no modification of the GluR2 ( $p=0.8481 ; n=5$ ) subunit of AMPA receptor. $\boldsymbol{H}$, Primary hippocampal neurons immunolabeling for GluR1 (green) and Shank (red). Neurons treated with TIMP-1 showed a statistically significant increase in GluR1/Shank colocalization $(p<0.005 ; n=10)$.

were bigger for spines smaller than the cutoff value, both groups showed a significant increase in spine head width 30 min after treatment with TAT-Pro compared with control and TAT-Alatreated neurons (width $<0.8 \mu \mathrm{m}: p<0.01$ among groups, $p<$ 0.01 TAT-Pro vs control, $p<0.05$ TAT-Pro vs TAT-Ala; width $\geq 0.8$ $\mu \mathrm{m}: p<0.0001$ among groups, $p<0.0001$ TAT-Pro vs control, $p<0.005$ TAT-Pro vs TAT-Ala). Furthermore, a significant increase in the percentage of growing spines $(p<0.01$ among groups, $p<0.05$ TAT-Pro vs control, $p<0.01$ TAT-Pro vs TATAla) and a corresponding decrease in the percentage of shrinking ones ( $p<0.01$ among groups, $p<0.05$ TAT-Pro vs control, $p<$ 0.01 TAT-Pro vs TAT-Ala) was detectable $30 \mathrm{~min}$ after treatment with TAT-Pro compared with control and TAT-Ala (Fig. 3D).
ADAM10 localization and activity influence subunit composition of synaptic AMPA receptors

$\mathrm{N}$-Cadherin regulates the number of AMPA receptors at synapses (Liao et al., 1999; Togashi et al., 2002; Kasai et al., 2003) and can interact with specific subunits of AMPA receptors (Dunah et al., 2005; Nuriya and Huganir, 2006; Saglietti et al., 2007). More recently, it has been shown that $\mathrm{N}$-cadherin inactivation results in fewer GluR1 synaptic clusters (Xie et al., 2008).

To test whether this N-cadherin-dependent modulation of AMPA receptor composition at synapses is associated with ADAM10-mediated N-cadherin cleavage, hippocampal slices from 3-week-old rats were incubated with TAT-Pro or TAT-Ala for $30 \mathrm{~min}$. As for primary neurons (Fig. 1), we first validated our 
A

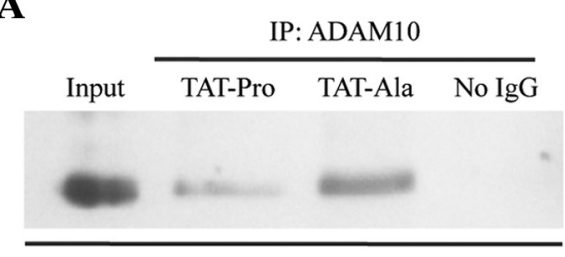

WB: SAP97

D

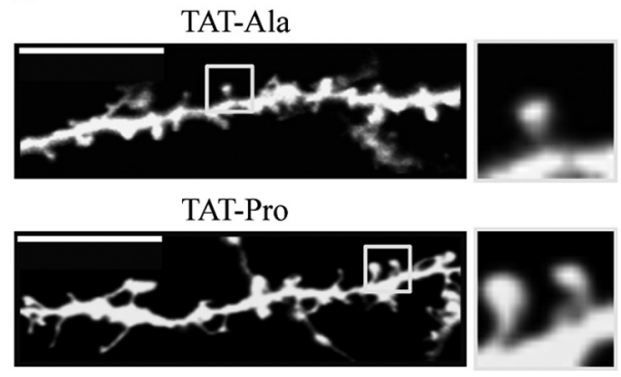

B

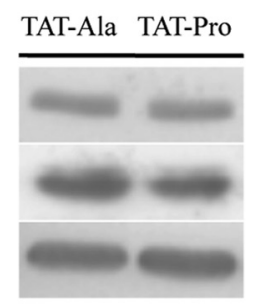

E

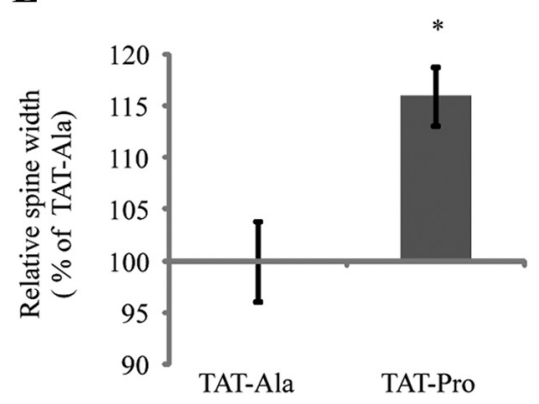

SAP97

ADAM10

Tubulin
C

TAT-Ala TAT-Pro

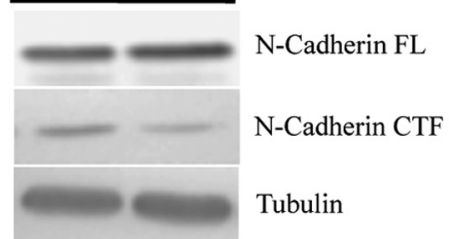

F

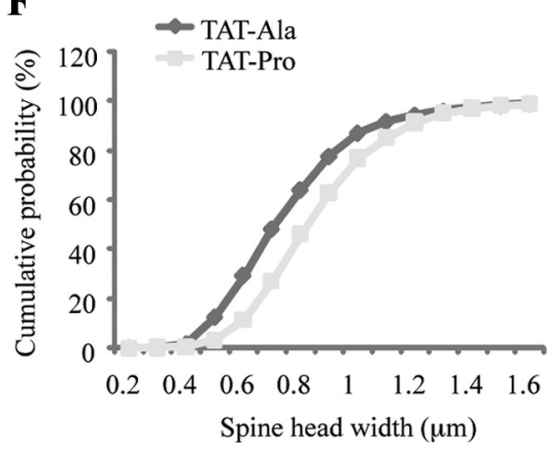

Figure 7. In vivo modulation of ADAM10/SAP97 interaction. A, TAT-Ala and TAT-Pro ADAM10 peptides were injected in vivo ( $3 \mathrm{nmol} / \mathrm{g}, 24 \mathrm{~h}$; 14 -d-old mice). Total homogenate was immunoprecipitated (IP) with an antibody against ADAM10, and Western blot (WB) analysis was performed with an antibody against SAP97. B, ADAM10 (TAT-Pro vs TAT-Ala, $p<0.05 ; 22$ animals from 3 independent experiments), but not SAP97 ( $p=0.0533 ; 22$ animals from 3 independent experiments), was decreased in the TIF purified from animals treated with TAT-Pro inhibitory peptide compared with animals treated with TAT-Ala. C, Western blot analysis performed in the TIF with antibodies against N-cadherin after $24 \mathrm{~h}$ in vivo treatment with TAT-Pro and TAT-Ala peptides. $\mathrm{N}$-cadherin FL/CTF ratio in TIF was statistically increased ( $p<0.05 ; 22$ animals from 3 independent experiments) in animals treated with TAT-Pro compared with animals treated with TAT-Ala. $D$, Morphological analysis of dendritic spines was conducted using a diolistic gene-gun system to propel Dil-coated particles into hippocampal sections of fixed brain tissue of TAT-Pro- and TAT-Alatreated mice. Scale bar, $10 \mu \mathrm{m}$. E, Diagram of average spine head width ( ${ }^{*} p<0.005$, TAT-Pro vs TAT-Ala; $n>900$ spines from 18 neurons from 4 different animals for each condition). $\boldsymbol{F}$, (umulative frequency plots from neurons treated with TAT-Ala and TAT-Pro ADAM10 peptides.

tool: in hippocampal slices, treatment with TAT-Pro decreased ADAM10 interaction with SAP97 (Fig. 4A) (TAT-Pro vs TATAla, $-59.5 \pm 9.9 \% ; p<0.005)$, decreased ADAM10 localization in the TIF (Fig. $4 B$ ) (TAT-Pro vs TAT-Ala, $-35.3 \pm 10.4 \%$; $p<$ 0.05 ) and, as a direct consequence, increased the $\mathrm{N}$-cadherin FL/ CTF ratio (Fig. 4C) (TAT-Pro vs TAT-Ala, $+53.9 \pm 16.4 \% ; p<$ $0.05)$.

In hippocampal slices treated with TAT-Pro, the GluR2 subunit of AMPA receptor (Fig. 4D), as well as all tested NMDA receptor subunits (Fig. $4 E$ ), did not show any modification of their localization in the postsynaptic compartment. Instead, GluR1 levels showed a specific and significant increase in TIF (Fig. $4 D$ ) (TAT-Pro vs TAT-Ala, $+30.7 \pm 3.2 \% ; p<0.0001$ ). This result was confirmed in hippocampal neurons treated with TAT-Pro where increased GluR1 localization in Shank-positive clusters was detectable (Fig. $4 F$ ) (TAT-Pro vs TAT-Ala, $+39.0 \pm$ $6.7 \% ; p<0.005)$. To demonstrate that the observed effect is dependent on $\mathrm{N}$-cadherin cleavage, we repeated GluR1/Shank colocalization assay in neurons transfected with GFP, with GFP$\mathrm{N}$-cadherin $\mathrm{Wt}$, or with the cleavage-defective GFP-N-cadherin GD construct. Statistical analysis revealed a significant increase in GluR1 levels in Shank-positive clusters in GFP-N-cadherin GD but not in GFP N-cadherin Wt transfected neurons (Fig. 4G) $(p<0.0002$ among groups, $p<0.002$ GD vs GFP, $p<0.0002 \mathrm{GD}$ vs Wt) compared with control.

\section{ADAM10 localization and activity influence synaptic AMPA receptor function}

Since interfering with ADAM10 activity at synapses leads to modification of the composition of AMPA receptors, we tested whether it is also capable of modifying AMPA receptor function. To this, we delivered postsynaptically nonpermeable Pro and Ala peptides (same effector sequence as TAT-Pro and TAT-Ala, respectively, but not bound to TAT-cargo domain) through the patch pipette while monitoring AMPA receptor-mediated EPSCs evoked by stimulation of Schaffer collaterals in CA1 pyramidal cells in the presence of bicuculline $(10 \mu \mathrm{M})$ and D-AP-5 $(50 \mu \mathrm{M})$. Thirty minutes after intracellular perfusion with Pro peptide, cells showed a significant increase in EPSC amplitudes compared with cells dialyzed with Ala peptide (Fig. $5 A, B)(p<0.0001)$. In a subset of experiments, we evaluated the contribution of AMPA receptors lacking the GluR2 subunit to the EPSCs measuring the $\mathrm{RI}$ at the beginning $(0 \mathrm{~min})$ and at the end $(30 \mathrm{~min})$ of the experiment (Fig. $5 A, C)($ at $-60,0$, and $+40 \mathrm{mV})$. RI values observed for both peptides at $0 \mathrm{~min}$ (Fig. $5 \mathrm{~A}, \mathrm{C}$ ) (Pro, $0.84 \pm 0.06$; Ala, $0.92 \pm 0.05)$ were similar to those previously observed at this age in CA1 pyramidal cells (Adesnik and Nicoll, 2007). No significant change in RI was observed in cells dialyzed for $30 \mathrm{~min}$ with Pro or Ala ADAM10 peptides (Fig. $5 A, C$ ) (Pro peptide, $p=0.4$; Ala peptide, $p=0.37$ ). Therefore, inhibition of ADAM10/SAP97 interaction not only induces a rise in GluR1 localization in the TIF but also increases the amplitude of AMPA receptor-mediated EPSCs, which do not appear to be mediated by an insertion of GluR2-lacking AMPA receptors.

Inhibition of ADAM10 activity by treatment with TIMP-1 results in decreased ADAM10-mediated N-cadherin processing and in a modification of AMPA receptors at synapses

To confirm the results obtained by using TAT-Pro peptide, we performed a pharmacological inhibition of ADAM10 activity by using TIMP-1 (Amour et al., 2000) in DIV10 hippocampal neurons.

As TAT-Pro peptide did, TIMP-1 treatment (15 nM, $30 \mathrm{~min}$ ) also caused an increase in N-cadherin FL (Fig. 6A) (TIMP-1 vs 
A

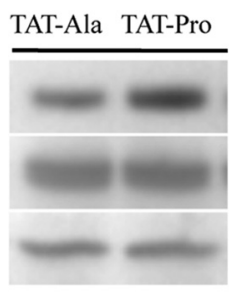

GluR1

\section{GluR2}

Tubulin

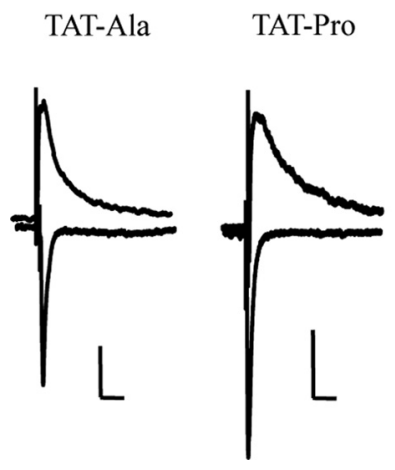

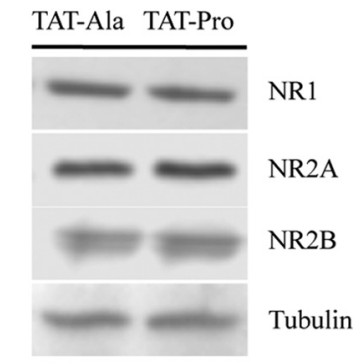

Tubulin

\section{D}

B

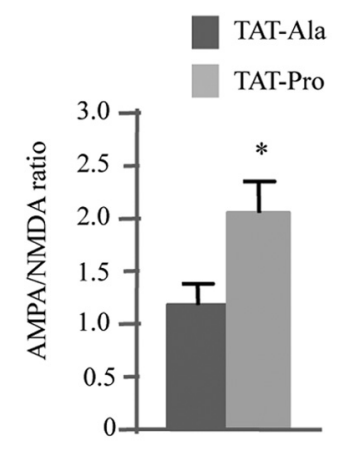

Figure 8. In vivo effect of ADAM10 synaptic activity on AMPA receptor composition and currents. $\boldsymbol{A}$, Western blot analysis performed in TIF with antibodies against GluR1 and GluR2 subunits of AMPA receptor; TAT-Pro increased the GluR1 subunit level ( $p<0.05 ; 22$ animals from 3 independent experiments), compared to animals treated with TAT-Ala control peptide. No change in synaptic abundance of GluR2 AMPA receptor subunit was found ( $p=0.8703 ; 22$ animals from 3 independent experiments) after $24 \mathrm{~h}$ in vivo treatment with TAT-Pro inhibitory peptide. $\boldsymbol{B}$, Western blot analysis performed on TIF with antibodies against NR1, NR2A, and NR2B subunits of NMDA receptor (NR1, $p=0.1700 ;$ NR2A, $p=0.6748 ; \mathrm{NR2B}, p=0.7424 ; 22$ animals from 3 independent experiments). $C$, Averaged EPSCs recorded at -80 and $+40 \mathrm{mV}$ from CA1 pyramidal cells from mice given injections, $24 \mathrm{~h}$ before slicing, of Tat-Ala and Tat-Pro ADAM10 peptides $(3 \mathrm{nmol} / \mathrm{g})$. Calibration: $50 \mathrm{pA}, 50 \mathrm{~ms}$. D, Bar graph summarizing the AMPA/ NMDA ratio obtained from mice treated with Tat-Ala or Tat-Pro peptide $\left({ }^{*} p<0.05\right.$; TAT-Pro, $2.05 \pm 0.29, n=13$; TAT-Ala, $1.18 \pm 0.19, n=14)$. Decay times of responses at $-80 \mathrm{mV}$ were $14.8 \pm 0.1$ and $14.5 \pm 0.1 \mathrm{~ms}$ in the TAT-Pro and TAT-Ala groups, respectively ( $p=$ 0.86); decay time responses of $-40 \mathrm{mV}$ were $135.5 \pm 10.1$ and $147.3 \pm 4.1 \mathrm{~ms}$ in the TAT-Pro and TAT-Ala groups, respectively $(n=13-14 ; p=0.26)$.

control, $+37.6 \pm 14.5 \% ; p<0.05)$, a concomitant decrease in $\mathrm{N}$-cadherin CTF (Fig. 6A) (TIMP-1 vs control, $-38.6 \pm 9.5 \%$; $p<0.01$ ), and a consequent increase in the FL/CTF ratio (Fig. $6 A$ ) (TIMP-1 vs control, $+131.5 \pm 23.7 \%$; $p<0.005$ ). As expected, TIMP-1 did not induce any modification of ADAM10 localization at synaptic sites, as demonstrated by both immunocytochemical analysis (Fig. $6 B)(p=0.3637)$ and Western blot analysis on TIF (Fig. 6C) $(p=0.2240)$. Furthermore, treatment with TIMP-1 significantly increased spine head width (Fig. $6 D, E)(p<0.0001)$, as confirmed by cumulative frequency plots analysis (Fig. $6 F$ ). As for TAT-Pro treatment, no changes in the mean spine density were found. Again, in TIMP-1-treated neurons, GluR1 levels were significantly increased in the postsynaptic membranes (Fig. 6G) (TIMP-1 vs control, $+32.2 \pm 5.3 \%$; $p<$ 0.0001 ), and GluR1 localization was higher in Shank-positive clusters compared with controls (Fig. 6H) (TIMP-1 vs control, $+48.9 \pm 7.6 \% ; p<0.005)$.

\section{In vivo modulation of ADAM10/SAP97 interaction}

The above-described results indicate that inhibition of ADAM10 activity in the postsynaptic compartment in primary hippocam- pal neurons and in acute hippocampal slices leads to an increase in the synaptic levels of N-cadherin FL and in spine head width and to a modification of AMPA receptor currents and composition at synapse.

To assess the role of ADAM10 activity in modulating longlasting rearrangements of glutamatergic synapse during development, we performed an in vivo treatment with TAT-Pro in young mice (2 weeks old). As shown in Figure 7A, $24 \mathrm{~h}$ after intraperitoneal administration of TAT-Pro ( $3 \mathrm{nmol} / \mathrm{g}$ ) (Marcello et al., 2007), ADAM10 interaction with SAP97 was reduced (Marcello et al., 2007) as well as the ADAM10 amount at synaptic sites (Fig. $7 B)$ (TAT-Pro vs TAT-Ala, $-34.7 \pm 4.4 \%$; $p<0.05$ ) with no modification of SAP97 levels (Fig. 7B). In addition, $24 \mathrm{~h}$ after administration of TAT-Pro, the N-cadherin FL/CTF ratio was increased at synapse (Fig. 7C) (TAT-Pro vs TAT-Ala, $+110.3 \pm$ $16.7 \%$; $p<0.05$ ), indicating a reduction in ADAM10-mediated $\mathrm{N}$-cadherin cleavage.

Morphological analysis of the size of dendritic spines was conducted using a diolistic gene gun system to propel DiI-coated particles into hippocampal sections of fixed brain tissue of TATPro- and TAT-Ala-treated mice (Fig. $7 D-F$ ). Statistical analysis revealed a significant increase in spine head width (Fig. $7 E)(p<$ 0.005 ) after treatment with TAT-Pro and cumulative frequency plots of spine head width confirmed the significant shift toward bigger spines (Fig. $7 F$ ). A significant decrease in the mean spine density was also found after in vivo TAT-Pro treatment (TATAla, $6.72 \pm 0.46$ spines $/ 10 \mu \mathrm{m}$; TAT-Pro, $5.53 \pm 0.31$ spines $/ 10$ $\mu \mathrm{m} ; p<0.05)$.

Moreover, mice treated with TAT-Pro peptide showed a significant increase in the levels of the GluR1 subunit of AMPA receptor (Fig. 8A) (TAT-Pro vs TAT-Ala, $+38.1 \pm 7.6 \%$; $p<$ 0.05 ) in the postsynaptic compartment when compared to TATAla-treated ones. No changes in synaptic abundance of GluR2 AMPA receptor subunit (Fig. $8 A$ ) and of NMDA receptor subunits (Fig. $8 B$ ) were found $24 \mathrm{~h}$ after in vivo treatment with TATPro. No modification of any tested protein was observed in the total homogenate (supplemental Fig. $3 A-C$, available at www. jneurosci.org as supplemental material).

To confirm that the above-described modification of GluR1 localization at synapse is mediated by the ADAM10/N-cadherin pathway and not by an a specific effect of TAT-Pro on SAP97 interaction with GluR1 (Gardoni et al., 2003; Waites et al., 2009), a coimmunoprecipitation assay was performed: mice treated with TAT-Pro showed no modification of the GluR1/SAP97 complex compared with animals treated with TAT-Ala (supplemental Fig. 4, available at www.jneurosci.org as supplemental material).

Again, as we did in vitro, we tested whether impairing ADAM10-mediated cleavage of $\mathrm{N}$-cadherins in vivo was accompanied by changes in synaptic currents. Fourteen-day-old mice were given intraperitoneal injections of either TAT-Pro or TATAla peptides ( $3 \mathrm{nmol} / \mathrm{g}$ ), and hippocampal slices were prepared $24 \mathrm{~h}$ later. AMPA and NMDA receptor-mediated EPSCs were recorded from CA1 pyramidal cells, respectively, at -80 and +40 $\mathrm{mV}$. The NMDA receptor-mediated contribution was measured $40 \mathrm{~ms}$ after initiation of the EPSC, a time point at which AMPA receptor-mediated EPSCs are absent or minimal. Treatment of mice with TAT-Pro induced a significant increase in the AMPA/ NMDA ratio compared with TAT-Ala treatment (Fig. $8 C, D$ ) $(p<0.05)$. No significant differences were observed in the decay times of EPSCs recorded at -80 and $+40 \mathrm{mV}$. 
A

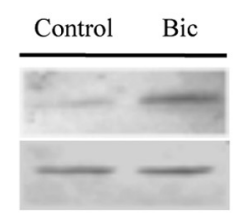

Actin
B

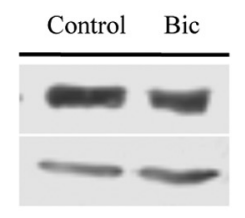

C

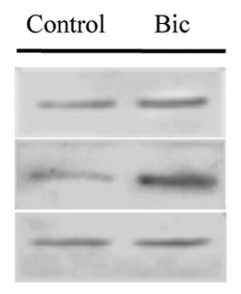

N-Cadherin FL

N-Cadherin CTF

Actin
D

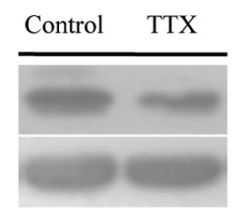

\section{ADAM10 \\ Tubulin}

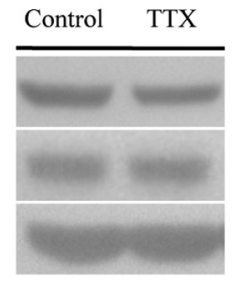

ADAM10

Tubulin

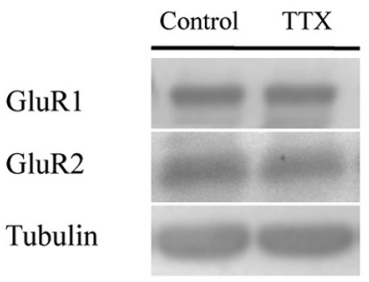

\section{$\mathrm{N}$-Cadherin FL \\ $\mathrm{N}-\mathrm{Cadherin} \mathrm{CTF}$}

Tubulin
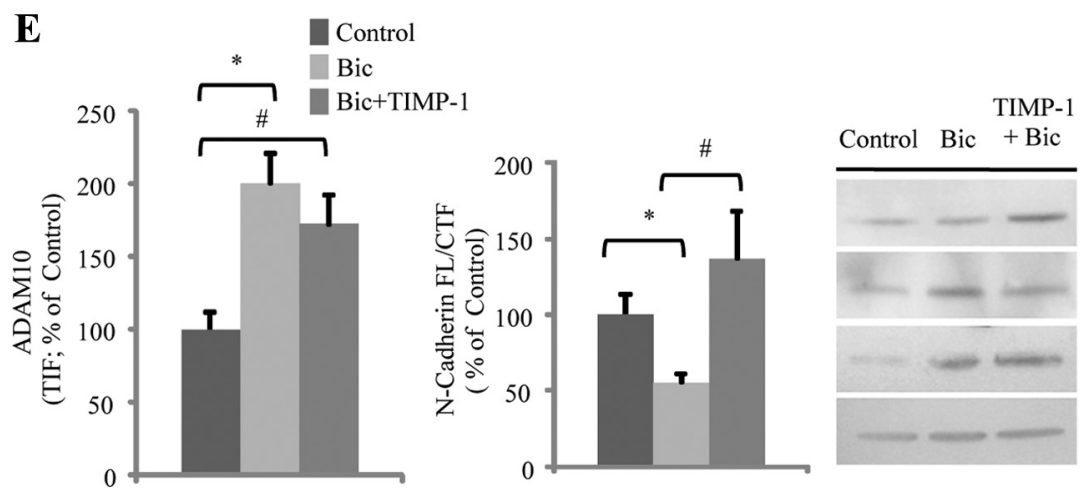

N-Cadherin FL

N-Cadherin CTF

ADAM10

Tubulin

\section{F}

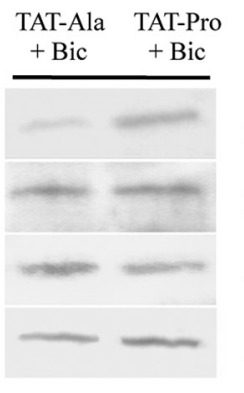

N-Cadherin FL

N-Cadherin CTF

ADAM10

Actin

Figure 9. Synaptic activity modulates ADAM10-mediated N-cadherin cleavage. A, Primary hippocampal neurons were stimulated or not with bicuculline (50 $\mu$ M, 30 min). Stimulation with bicuculline increased ADAM10 amount in the TIF ( $p<0.005 ; n=13)$. $\boldsymbol{B}$, Surface assay performed with the cross-linker BS ${ }^{3}$, a membrane-impermeable amine-reactive cross-linker reagent, on primary hippocampal neurons after stimulation with bicuculline ( $50 \mu \mathrm{M}, 30 \mathrm{~min}$ ). Western blot analysis shows a significant decrease in the intracellular amount of $A D A M 10$ ( $p<0.05 ; n=3$ ) in neurons treated with bicuculline compared with control cells; all data have been normalized using tubulin. ADAM10 surface pool was not detectable because of the formation of high-molecularweight aggregates that did not enter the gel. C, Stimulation with bicuculline (50 $\mu \mathrm{m}, 30 \mathrm{~min}$ ) induced a statistically significant decrease in the $\mathrm{N}$-cadherin FL/CTF ratio ( $p<0.00005 ; n=13$ ). $\boldsymbol{D}$, Western blot analysis performed in TIF after treatment with TTX ( $2 \mu \mathrm{M}, 30 \mathrm{~min})$; TTX treatment induced a statistically significant decrease in ADAM10 amount ( $p<0.01 ; n=7)$ but no modification of GluR1, GluR2 and the N-cadherin FL/CTF ratio. $\boldsymbol{E}$, Primary hippocampal neurons were incubated or not with the ADAM10-specific inhibitor peptide TIMP-1 (15 nM, $1 \mathrm{~h}$ ) and stimulated with bicuculline (50 $\mu \mathrm{M}, 30 \mathrm{~min}$ ); untreated cells were used as the control. Neurons pretreated or not with TIMP-1 and stimulated with bicuculline showed a statistically significant increased amount of ADAM10 in the TIF compared with controls ( $p<0.01$ among groups; ${ }^{*} p<0.005$, bicuculline vs control; ${ }^{*} p<0.05$, TIMP-1 plus bicuculline vs control; $n=7$ ). Neurons stimulated with bicuculline showed statistically significant modification of the $\mathrm{N}$-cadherin $\mathrm{FL} / \mathrm{CTF}$ ratio ( $p<0.05$ among groups; ${ }^{*} p<0.0005$, bicuculline vs control; ${ }^{\#} p<0.05$, TIMP- 1 plus bicuculline vs bicuculline; $n=7$; one-way ANOVA, followed by Bonferroni's as a post hoc comparison test) compared with control and TIMP-1-treated cells. F, Western blot analysis performed in TIF with antibodies against ADAM10 and the C-terminal region of $\mathrm{N}$-cadherin. Primary hippocampal neurons (DIV10) were incubated with both TAT-Ala and TAT-Pro ADAM10 peptides for 2 min and stimulated with bicuculline (50 $\mu$, $30 \mathrm{~min}$ ). Neurons pretreated with TAT-Pro inhibitory peptide showed a statistically significant decrease in ADAM10 localization in the TIF ( $p<0.005$, TAT-Pro vs TAT-Ala; $n=5)$ and a consequent increase in the $\mathrm{N}$-cadherin FL/CTF ratio ( $p<0.05$, TAT-Pro vs TAT-Ala; $n=5)$ compared with TAT-Ala pretreated cells. Bic, Bicuculline.

\section{Synaptic activity modulates ADAM10-mediated $\mathrm{N}$-cadherin cleavage}

We demonstrated in vitro and in vivo that, under basalunstimulated conditions, inhibition of ADAM10-dependent $\mathrm{N}$-cadherin cleavage leads to a specific and long-lasting modification of the morphology of the glutamatergic synapse as well as of AMPA receptor subunit composition and function.

We then asked whether these mechanisms could be modulated by synaptic activity. To this, DIV10 hippocampal neurons were treated with bicuculline $(50 \mu \mathrm{M}, 30 \mathrm{~min})$. After treatment, TIF was purified and analyzed by Western blot. As shown in Figure $9 A$, neurons stimulated with bicuculline showed a significant increase in ADAM10 levels in the TIF (bicuculline vs control, $+71.0 \pm 20.5 \%$; $p<0.005)$.

Since ADAM10 is active only when inserted into the postsynaptic membrane, we performed a surface assay with the crosslinker $\mathrm{BS}^{3}$ after treatment with bicuculline: in lysates of neurons treated with bicuculline, the intracellular amount of ADAM10 was decreased compared with control cells (Fig. 9B) (bicuculline vs control, $-36.3 \pm 6.2 \% ; p<0.05)$, indicating an increased insertion of the enzyme into the membrane. As expected, the increased ADAM10 localization in the postsynaptic membrane was associated with a decrease in the N-cadherin FL/CTF ratio (Fig. 9C) (bicuculline vs control, $-41.4 \pm 6.7 \%$; $p<0.00005$ ). On the other hand, treatment with TTX $(2 \mu \mathrm{M}, 30 \mathrm{~min})$, a neural activity blocker, led to decreased ADAM10 levels at synapses (Fig. 9D) (TTX vs control, $-31.3 \pm 7.4 \% ; p<0.01$ ) that were not paralleled by any modification of the N-cadherin FL/CTF ratio and GluR1 levels at synapses (Fig. 9D). Moreover, $3 \mathrm{~h}$ TTX treatment did not result in any modification of ADAM10 levels at synaptic sites as well as of N-cadherin and GluR1 (see also Sutton et al., 2006; Turrigiano et al., 1998), suggesting that TTX induces only a transient effect on ADAM10 localization at synaptic sites not sufficient to affect the N-cadherin/GluR1 pathway (data not shown). 

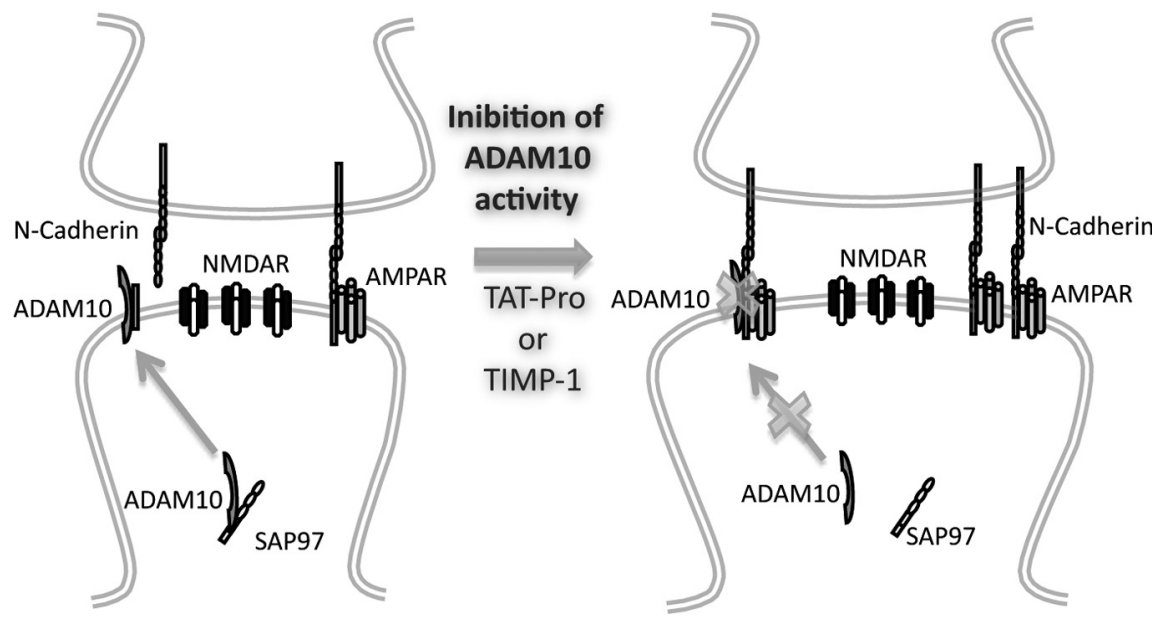

Figure 10. Diagrammatic representation of the complex sequence of events through which inhibition of ADAM10-dependent $\mathrm{N}$-cadherin cleavage, by treatment with TAT-Pro or TIMP-1, affects spine maturation and controls structure and function of glutamatergic synapses.

\section{ADAM10 activity is needed for activity-dependent $\mathrm{N}$-cadherin processing}

To verify whether the above-described bicuculline-induced $\mathrm{N}$-cadherin cleavage in neurons was mediated by ADAM10, bicuculline treatment was repeated in the presence or absence of TIMP-1. DIV10 hippocampal neurons were treated with TIMP-1 (15 nM, $1 \mathrm{~h}$ ) and stimulated with bicuculline (50 $\mu \mathrm{M}, 30 \mathrm{~min})$. As expected, stimulation with bicuculline increased ADAM10 localization to the postsynaptic compartment in the absence and in the presence of TIMP-1 (Fig. $9 E)(p<0.01$ among groups; bicuculline vs control, $+108.6 \pm 24.2 \%, p<0.005$; TIMP-1, + bicuculline vs control, $+72.0 \pm 19.2 \%, p<0.05)$, but pretreatment with TIMP-1 prevented the increase of ADAM10-mediated $\mathrm{N}$-cadherin cleavage induced by bicuculline (Fig. 9E) (FL/CTF: $p<0.05$ among groups; bicuculline vs control, $-40.4 \pm 11.3 \%$, $p<0.0005$; TIMP-1, + bicuculline vs bicuculline, $+134.6 \pm$ $40.6 \%, p<0.05)$. Thus, TIMP-1 blocks activity-dependent cleavage of $\mathrm{N}$-cadherin mediated by ADAM10 without affecting ADAM10 subcellular localization.

Since bicuculline increases ADAM10 localization to the postsynaptic membrane and its activity on $\mathrm{N}$-cadherin and since ADAM10 localization in TIF is regulated by SAP97, we asked whether SAP97 was involved in the bicuculline-dependent regulation of ADAM10 cleavage on N-cadherin. To this, primary hippocampal neurons (DIV10) were pretreated with TAT-Pro or TAT-Ala $(2 \mathrm{~min})$ and stimulated with bicuculline $(50 \mu \mathrm{M}, 30$ $\mathrm{min})$. As shown in Figure 9F, TAT-Pro treatment reduced ADAM10 (TAT-Pro vs TAT-Ala, $-45.6 \pm 7.5 \% ; p<0.005$ ) localization in TIF also in neurons pretreated with bicuculline. Accordingly, the reduced ADAM10 localization in the postsynaptic membrane was associated with an enhanced $\mathrm{N}$-cadherin FL/CTF ratio (Fig. 9F) (TAT-Pro vs TAT-Ala, $+103.2 \pm 33.5 \%$; $p<0.05$ ). These results indicate the binding to SAP97 as necessary also for activitydependent localization of ADAM10 at synaptic sites.

\section{Discussion}

Here we demonstrate that synaptic activity of an enzyme belonging to the disintegrin and metalloproteinase family, ADAM10, represents a new way to regulate the number and function of AMPA receptors at hippocampal excitatory synapses (Fig. 10). Interfering with ADAM10 localization at synapses is sufficient to induce a significant decrease in ADAM10-mediated $\mathrm{N}$-cadherin cleavage, leading to accumulation of $\mathrm{N}$-cadherin FL. This molecular event correlates with an increase in spine head width and with a modification of AMPA receptor currents and subunit composition both in vitro and in vivo.

The existence of a close coordination among $\mathrm{N}$-cadherin, spine size, and AMPA receptor content has been put forward: $\mathrm{N}$ cadherin activation results in more AMPA receptors and, consequently, in bigger and stable synapses (Kasai et al., 2003; Kopec et al., 2007; Xie et al., 2008). Here we show that ADAM10-dependent cleavage of N-cadherin represents an upstream event of these pathways. Inhibition of ADAM10-mediated processing of $\mathrm{N}$-cadherin induces a significant increase in spine head width in both small-immature and large-mature spines, as observed within $30 \mathrm{~min}$ in time-lapse experiments. Interestingly, spine enlargement starts $18 \mathrm{~min}$ after stabilization of $\mathrm{N}$-cadherin FL and remains stable up to 30 min after ADAM10 inhibition.

Notably, these observations have been confirmed by in vivo experiments. The TAT-Pro peptide used in the present study is a useful tool not only for in vitro but also for in vivo studies. This is of relevance because in vivo study of $\mathrm{N}$-cadherin processing was formerly hampered by the lack of sensitive tools. In particular, here we show that the in vivo effect of ADAM10 inhibition on spine morphology is quite long-lasting and stable, being significant still $24 \mathrm{~h}$ after treatment.

It has been shown that accumulation of $\mathrm{N}$-cadherin FL results in more GluR1 synaptic clusters, whereas N-cadherin inactivation results in less GluR1 clusters (Xie et al., 2008). Accordingly, our results show that 30 min after stabilization of the full-length form of N-cadherin, a significant recruitment of GluR1 into the postsynaptic compartment is detectable. No significant changes in the RI were observed in cells dialyzed with ADAM10 inhibitory peptide, suggesting the absence of any significant modification in the percentage of GluR2 containing AMPA receptors. This is only an apparent discrepancy between molecular and electrophysiological analysis; in fact, molecular analysis performed in the TIF allows detecting modifications of AMPA receptor subunits' levels at synaptic sites but does not give information on modifications of AMPA receptor subunit insertion/endocytosis taking place within the synaptic compartment. Moreover, the observed selective GluR1 augmentation (about 30\%) could simply not lead to a detectable change in RI that can be observed only in the presence of homomeric GluR1 receptor.

Long-term persistence of the effect of ADAM10 inhibition on synaptic GluR1-containing AMPA receptors and on AMPA receptor currents was demonstrated by $24 \mathrm{~h}$ in vivo experiments, again confirming the close correlation between spine size and GluR1 levels.

The major issue of the present study is to analyze molecular, functional, and morphological outcomes of the ADAM10/Ncadherin pathway. Recent studies provided indications that ADAM10 is responsible for the first step of the proteolytic processing of N-cadherin, leading to the generation of the CTF1 fragment (Reiss et al., 2005; Uemura et al., 2006). In the present study, we show that transfection of hippocampal neurons with the cleavage-defective $\mathrm{N}$-cadherin construct, mutated in the 
ADAM10 cleavage site, induces enlargement of spine size not observed after transfection with $\mathrm{N}$-cadherin $\mathrm{Wt}$, possibly by adding at the cell membrane a new pool of N-cadherin that could not be cleaved by ADAM10, thus altering the N-cadherin FL/CTF ratio.

It is noteworthy that ADAM10 is responsible for both constitutive and activity-dependent $\mathrm{N}$-cadherin cleavage. Treatment with bicuculline shows that it is possible to increase ADAM10 synaptic localization and, consequently, its activity toward $\mathrm{N}$-cadherin on neuronal activity. Furthermore, both TAT-Pro peptide and TIMP-1 block in vitro ADAM10 activity stimulated by neuronal activity. Overall, these results confirm the already proposed role for $\mathrm{N}$-cadherin in homeostatic plasticity (Peng et al., 2009), suggesting that ADAM10-dependent N-cadherin cleavage could represent a new modality regulating the strength of the excitatory synpase.

This is not the first report showing that a molecular and functional connection between the ADAM family/adhesion molecules and scaffolding elements of the PSD-MAGUK family (Fukata et al., 2006) leads to modifications of AMPA receptormediated currents. Thus, since several types of adhesion molecules and PSD-MAGUKs, with redundant functions, are present at synapses, it is likely that functional cross talks between these key families of synaptic proteins are relevant to control synaptic maturation and stabilization in vivo. We have also previously shown such a functional correlation between PSD-MAGUKs and the ADAM family, describing a direct interaction between ADAM10 and the SAP97 member of the PSD-MAGUK family (Marcello et al., 2007).

Here we have cleared up the morphological and functional outcomes of this interaction on the glutamatergic synapse, demonstrating an important role for ADAM10 in the complex and coordinated sequence of events through which $\mathrm{N}$-cadherin affects spine maturation.

\section{References}

Aarts M, Liu Y, Liu L, Besshoh S, Arundine M, Gurd JW, Wang YT, Salter MW, Tymianski M (2002) Treatment of ischemic brain damage by perturbing NMDA receptor-PSD-95 protein interactions. Science 298:846-850.

Abe K, Chisaka O, Van Roy F, Takeichi M (2004) Stability of dendritic spines and synaptic contacts is controlled by alpha $\mathrm{N}$-catenin. Nat Neurosci 7:357-363.

Adesnik H, Nicoll RA (2007) Conservation of glutamate receptor 2-containing AMPA receptors during long-term potentiation. J Neurosci 27:4598-4602.

Amour A, Knight CG, Webster A, Slocombe PM, Stephens PE, Knäuper V, Docherty AJ, Murphy G (2000) The in vitro activity of ADAM-10 is inhibited by TIMP-1 and TIMP-3. FEBS Lett 473:275-279.

Bamji SX (2005) Cadherins: actin with the cytoskeleton to form synapses. Neuron 47:175-178.

Benson DL, Tanaka H (1998) N-Cadherin redistribution during synaptogenesis in hippocampal neurons. J Neurosci 18:6892-6904.

Boggon TJ, Murray J, Chappuis-Flament S, Wong E, Gumbiner BM, Shapiro L (2002) C-cadherin ectodomain structure and implications for cell adhesion mechanisms. Science 296:1308-1313.

Dingledine R, Borges K, Bowie D, Traynelis SF (1999) The glutamate receptor ion channels. Pharmacol Rev 51:7-61.

Dunah AW, Hueske E, Wyszynski M, Hoogenraad CC, Jaworski J, Pak DT, Simonetta A, Liu G, Sheng M (2005) LAR receptor protein tyrosine phosphatases in the development and maintenance of excitatory synapses. Nat Neurosci 8:458-467.

Fahrenholz F, Postina R (2006) Alpha-secretase activation-an approach to Alzheimer's disease therapy. Neurodegener Dis 3:255-261.

Fukata Y, Adesnik H, Iwanaga T, Bredt DS, Nicoll RA, Fukata M (2006) Epilepsy-related ligand/receptor complex LGI1 and ADAM22 regulate synaptic transmission. Science 313:1792-1795.
Gardoni F, Bellone C, Cattabeni F, Di Luca M (2001) Protein kinase C activation modulates alpha-calmodulin kinase II binding to NR2A subunit of N-methyl-D-aspartate receptor complex. J Biol Chem 276:7609-7613.

Gardoni F, Mauceri D, Fiorentini C, Bellone C, Missale C, Cattabeni F, Di Luca M (2003) CaMKII-dependent phosphorylation regulates SAP97/ NR2A interaction. J Biol Chem 278:44745-44752.

Gardoni F, Marcello E, Di Luca M (2009) PSD-MAGUK proteins and their role in central nervous system disorders. Neuroscience 158:324-333.

Greger IH, Ziff EB, Penn AC (2007) Molecular determinants of AMPA receptor subunit assembly. Trends Neurosci 30:407-416.

Hall RA, Soderling TR (1997) Quantitation of AMPA receptor surface expression in cultured hippocampal neurons. Neuroscience 78:361-371.

Janes PW, Saha N, Barton WA, Kolev MV, Wimmer-Kleikamp SH, Nievergall E, Blobel CP, Himanen JP, Lackmann M, Nikolov DB (2005) Adam meets Eph: an ADAM substrate recognition module acts as a molecular switch for ephrin cleavage in trans. Cell 123:291-304.

Kasai H, Matsuzaki M, Noguchi J, Yasumatsu N, Nakahara H (2003) Structure-stability-function relationships of dendritic spines. Trends Neurosci 26:360-368.

Kopec CD, Real E, Kessels HW, Malinow R (2007) GluR1 links structural and functional plasticity at excitatory synapses. J Neurosci 27:13706-13718.

Lau CG, Zukin RS (2007) NMDA receptor trafficking in synaptic plasticity and neuropsychiatric disorders. Nat Rev Neurosci 8:413-426.

Liao D, Zhang X, O’Brien R, Ehlers MD, Huganir RL (1999) Regulation of morphological postsynaptic silent synapses in developing hippocampal neurons. Nat Neurosci 2:37-43.

Marcello E, Gardoni F, Mauceri D, Romorini S, Jeromin A, Epis R, Borroni B, Cattabeni F, Sala C, Padovani A, Di Luca M (2007) Synapse-associated protein-97 mediates alpha-secretase ADAM10 trafficking and promotes its activity. J Neurosci 27:1682-1691.

Mauceri D, Cattabeni F, Di Luca M, Gardoni F (2004) Calcium/ calmodulin-dependent protein kinase II phosphorylation drives synapseassociated protein 97 into spines. J Biol Chem 279:23813-23821.

Mendez P, De Roo M, Poglia L, Klauser P, Muller D (2010) N-cadherin mediates plasticity-induced long-term spine stabilization. J Cell Biol 189:589-600.

Newpher TM, Ehlers MD (2008) Glutamate receptor dynamics in dendritic microdomains. Neuron 58:472-497.

Nuriya M, Huganir RL (2006) Regulation of AMPA receptor trafficking by N-Cadherin. J Neurochem 97:652-661.

Nusser Z (2000) AMPA and NMDA receptors: similarities and differences in their synaptic distribution. Curr Opin Neurobiol 10:337-341.

Okamura K, Tanaka H, Yagita Y, Saeki Y, Taguchi A, Hiraoka Y, Zeng LH, Colman DR, Miki N (2004) Cadherin activity is required for activityinduced spine remodeling. J Cell Biol 167:961-972.

Okuda T, Yu LM, Cingolani LA, Kemler R, Goda Y (2007) beta-Catenin regulates excitatory postsynaptic strength at hippocampal synapses. Proc Natl Acad Sci U S A 104:13479-13484.

Peng YR, He S, Marie H, Zeng SY, Ma J, Tan ZJ, Lee SY, Malenka RC, Yu X (2009) Coordinated changes in dendritic arborization and synaptic strength during neural circuit development. Neuron 61:71-84.

Piccoli G, Verpelli C, Tonna N, Romorini S, Alessio M, Nairn AC, Bachi A, Sala C (2007) Proteomic analysis of activity-dependent synaptic plasticity in hippocampal neurons. J Proteome Res 6:3203-3215.

Pinheiro P, Mulle C (2006) Kainate receptors. Cell Tissue Res 326:457-482.

Reiss K, Maretzky T, Ludwig A, Tousseyn T, de Strooper B, Hartmann D, Saftig P (2005) ADAM10 cleavage of N-Cadherin and regulation of cellcell adhesion and beta-catenin nuclear signalling. EMBO J 24:742-752.

Reiss K, Maretzky T, Haas IG, Schulte M, Ludwig A, Frank M, Saftig P (2006) Regulated ADAM10-dependent ectodomain shedding of gamma-protocadherin C3 modulates cell-cell adhesion. J Biol Chem 281:21735-21744.

Saglietti L, Dequidt C, Kamieniarz K, Rousset MC, Valnegri P, Thoumine O, Beretta F, Fagni L, Choquet D, Sala C, Sheng M, Passafaro M (2007) Extracellular interactions between GluR2 and N-Cadherin in spine regulation. Neuron 54:461-477.

Sala C, Piëch V, Wilson NR, Passafaro M, Liu G, Sheng M (2001) Regulation of dendritic spine morphology and synaptic function by Shank and Homer. Neuron 31:115-130.

Sutton MA, Ito HT, Cressy P, Kempf C, Woo JC, Schuman EM (2006) Min- 
iature neurotransmission stabilizes synaptic function via tonic suppression of local dendritic protein synthesis. Cell 125:785-799.

Tai CY, Kim SA, Schuman EM (2008) Cadherins and synaptic plasticity. Curr Opin Cell Biol 20:567-575.

Togashi H, Abe K, Mizoguchi A, Takaoka K, Chisaka O, Takeichi M (2002) Cadherin regulates dendritic spine morphogenesis. Neuron 35:77-89.

Turrigiano GG, Leslie KR, Desai NS, Rutherford LC, Nelson SB (1998) Activity-dependent scaling of quantal amplitude in neocortical neurons. Nature 391:892-896.

Uemura K, Kihara T, Kuzuya A, Okawa K, Nishimoto T, Ninomiya H, Sugimoto H, Kinoshita A, Shimohama S (2006) Characterization of sequential N-Cadherin cleavage by ADAM10 and PS1. Neurosci Lett 402:278-283.
Waites CL, Specht CG, Härtel K, Leal-Ortiz S, Genoux D, Li D, Drisdel RC, Jeyifous O, Cheyne JE, Green WN, Montgomery JM, Garner CC (2009) Synaptic SAP97 isoforms regulate AMPA receptor dynamics and access to presynaptic glutamate. J Neurosci 29:4332-4345.

Xie Z, Photowala H, Cahill ME, Srivastava DP, Woolfrey KM, Shum CY, Huganir RL, Penzes P (2008) Coordination of synaptic adhesion with dendritic spine remodeling by AF-6 and kalirin-7. J Neurosci 28:6079-6091.

Yu X, Malenka RC (2003) Beta-catenin is critical for dendritic morphogenesis. Nat Neurosci 6:1169-1177.

Zito K, Scheuss V, Knott G, Hill T, Svoboda K (2009) Rapid functional maturation of nascent dendritic spines. Neuron 61:247-258. 